\title{
1 A novel heteromeric pantothenate kinase complex 2 in apicomplexan parasites
}

Erick T. Tjhin ${ }^{1 \dagger}$, Vanessa M. Howieson ${ }^{1 \dagger}$, Christina Spry ${ }^{1}$, Giel G. van Dooren ${ }^{1}$ and Kevin J. Saliba ${ }^{1,2, *}$

1. Research School of Biology, The Australian National University, Canberra, Australia

2. Medical School, The Australian National University, Canberra, Australia

*To whom correspondence should be addressed: kevin.saliba@anu.edu.au

†These authors contributed equally to this work. 


\section{ABSTRACT}

Coenzyme $A$ is synthesised from pantothenate via five enzyme-mediated steps. The first step is catalysed by pantothenate kinase (PanK). All PanKs characterised to date form homodimers. Many organisms express multiple PanKs. In some cases, these PanKs are not functionally redundant, and some appear to be non-functional. Here, we investigate the PanKs in two pathogenic apicomplexan parasites, Plasmodium falciparum and Toxoplasma gondii. Each of these organisms express two PanK homologues (PanK1 and PanK2). We demonstrate that PfPanK1 and PfPanK2 associate, forming a single, functional PanK complex that includes the multi-functional protein, Pf14-3-3I. Similarly, we demonstrate that TgPanK1 and TgPanK2 form a single complex that possesses PanK activity. Both TgPanK1 and TgPanK2 are essential for T. gondii proliferation, specifically due to their PanK activity. Our study constitutes the first examples of heteromeric PanK complexes in nature and provides an explanation for the presence of multiple PanKs within certain organisms.

\section{INTRODUCTION}

Coenzyme A (CoA) is an essential enzyme cofactor in all living organisms ${ }^{1}$. CoA itself, and the CoA-derived phosphopantetheine prosthetic group required by various carrier proteins, function as acyl group carriers and activators in key cellular processes such as fatty acid biosynthesis, $\beta$-oxidation and the citric acid cycle. Pantothenate kinase (PanK) catalyses the first step in the conversion of pantothenate (vitamin $B_{5}$ ) to CoA 2 . PanKs are categorised into three distinct types, type I, II and III based on their primary sequences, structural fold, enzyme kinetics and inhibitor sensitivity. PanKs from all three types have been shown to exist as homodimers based on their solved protein structures ${ }^{3-10}$. All eukaryotic PanKs that have been characterised so far are type II PanKs ${ }^{5}$. Interestingly, many eukaryotes express multiple PanKs (such as Arabidopsis thaliana ${ }^{11,12}$, Mus musculus ${ }^{13-16}$ and Homo sapiens 17-21), and in some cases it is clear that these PanKs are not functionally redundant 15,22. For example, mutations in only one of four type II PanKs in humans causes a neurodegenerative disorder known as PanK-associated neurodegeneration ${ }^{17}$. Some bacteria also express multiple PanKs. For example, some Mycobacterium ${ }^{23}$, Streptomyces ${ }^{7}$ and Bacillus ${ }^{7,24,25}$ species have both type I and type III PanKs, while a select few bacilli (including the category A biodefense pathogen Bacillus anthracis) carry both a type II and type III PanK ${ }^{7}$. In some organisms harbouring multiple PanKs, it has not been possible to demonstrate functional activity for all enzymes. One of the four human type II PanKs is shown to be catalytically inactive ${ }^{2126}$, as is a type III PanK from Mycobacterium tuberculosis ${ }^{23}$, and a type II PanK 
52 from $B$. anthracis ${ }^{7}$. The reason for the presence of multiple PanKs within certain cells, and

53 the apparent inactivity of certain PanKs, is unclear.

Two putative genes coding for PanK enzymes have been identified in each of the genomes of the pathogenic apicomplexan parasites Plasmodium falciparum (PF3D7_1420600 (Pfpank1) and PF3D7_1437400 (Pfpank2)) and Toxoplasma gondii (TGME49_307770 (Tgpank1) and TGME49_235478 (Tgpank2)). We have recently shown that mutations in PfPanK1 alter PanK activity in P. falciparum, providing evidence that PfPanK1 is an active PanK, at least in the disease-causing stage of the parasite's lifecycle ${ }^{27}$. The function of PfPanK2, and its contribution to PanK activity in $P$. falciparum, is unknown. PfPanK2 contains a unique, large insert in a loop associated with the dimerisation of PanKs in their native conformation ${ }^{8}$ and this may affect its ability to form a dimer, rendering it inactive ${ }^{28}$. No functional information is available on the putative $T$. gondii PanKs, but a genome-wide CRISPR-Cas9 screen of the T. gondii genome predicted that both PanK genes are important for parasite growth in vitro ${ }^{29}$. Similarly, a recent genome-wide insertional mutagenesis study of $P$. falciparum has predicted both PfPanK1 and PfPanK2 to be essential ${ }^{30}$. These results suggest that the PanK2 proteins of these parasites play important role(s), although their exact function remains unclear.

In this study, we demonstrate that PanK1 and PanK2 from P. falciparum and T. gondii are part of the same, multimeric protein complex. This constitutes the first identification of a heteromeric PanK complex in nature. Furthermore, our data provide the first evidence that PanK2 is essential for PanK function in apicomplexans.

\section{RESULTS}

\section{PfPanK1 and PfPanK2 are part of the same protein complex}

The importance and role of PfPanK2 in apicomplexan parasites have not previously been established. To characterise the $P$. falciparum PanK2 homologue (PfPanK2), we first determined where in the parasite the protein localises. We episomally expressed PfPanK2-

81 GFP in asexual blood stage $P$. falciparum parasites and found that PfPanK2-GFP is 82 localised throughout the parasite cytosol and is not excluded from the nucleus (Figure 1a). 83 This is a similar localisation to what we observed for PfPanK1-GFP previously ${ }^{27}$. Western blotting of proteins separated by SDS-PAGE revealed that PfPanK2-GFP has a molecular mass consistent with the predicted mass of the fusion protein $(\sim 118 \mathrm{kDa}$; Figure $1 \mathbf{b})$, which 
86 is slightly higher than the predicted mass of PfPanK1-GFP ( 87 kDa; Figure 1b). As PanKs

87 from other organisms exist as homodimers, we undertook blue native-PAGE to determine 88 whether PfPanK1-GFP and PfPanK2-GFP exist in protein complexes. Interestingly, under 89 native conditions, both PfPanK1-GFP and PfPanK2-GFP were found to be part of 90 complexes that are $\sim 240 \mathrm{kDa}$ in mass (Figure 1b).

92 To determine the activity and protein composition of these complexes, we set out to purify 93 the PfPanK1-GFP and PfPanK2-GFP complexes by immunoprecipitation. As a control, we 94 also purified untagged GFP. We verified that most of the GFP-tagged proteins were captured from the total lysates prepared from the different cell lines, with bands corresponding to PfPanK1-GFP, PfPanK2-GFP and the untagged GFP epitope tag detected in the bound fraction of the respective cell lines (Figure S1). To determine whether the purified PfPanK1 and PfPanK2 complexes possess PanK activity, we performed a $\left[{ }^{14} \mathrm{C}\right]$ pantothenate phosphorylation assay. We found that $50-60 \%$ of the $\left[{ }^{14} \mathrm{C}\right]$ pantothenate initially present in the reaction was phosphorylated within $90 \mathrm{~min}$ by the immunopurified complex from both the PfPanK1-GFP- and PfPanK2-GFP-expressing lines (Figure 1c).

102 Conversely, the immunopurified untagged GFP did not display PanK activity (Figure 1c). 103 These experiments provide the first indication that PfPanK1 and PfPanK2 are part of an 104 active PanK enzyme complex in $P$. falciparum parasites. They also provide the first indication that PfPanK2 contributes to PanK activity in these parasites. 


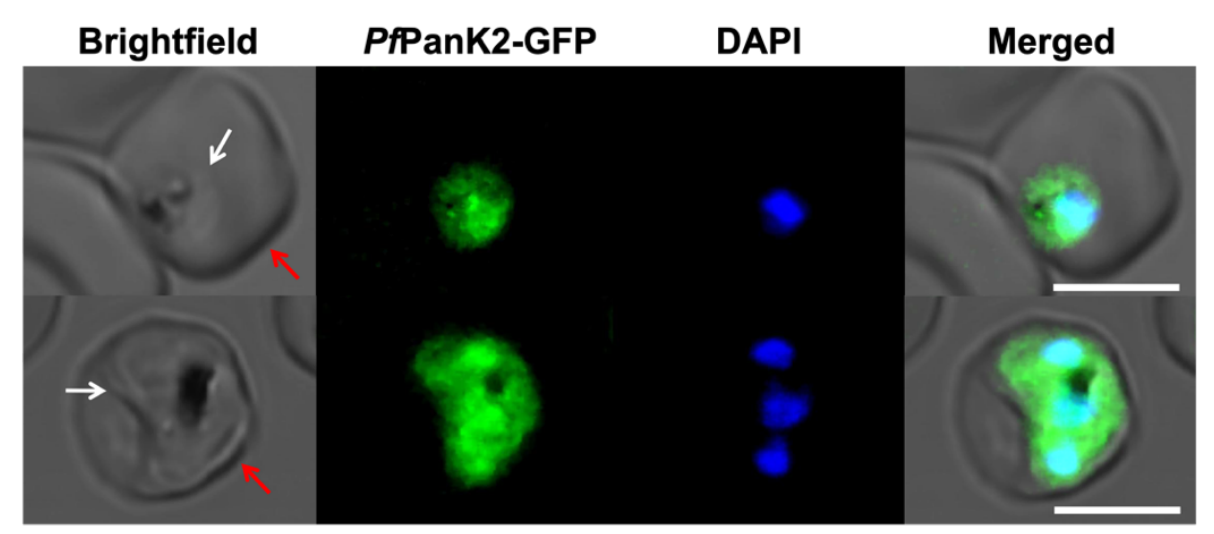

b

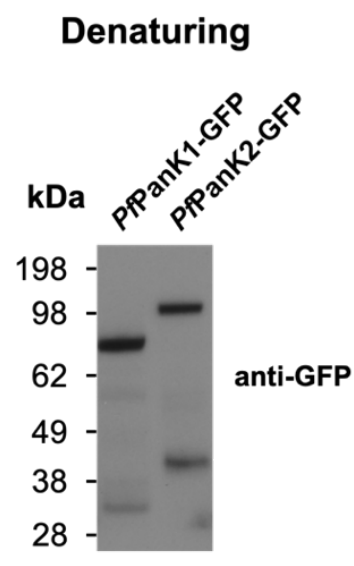

Native

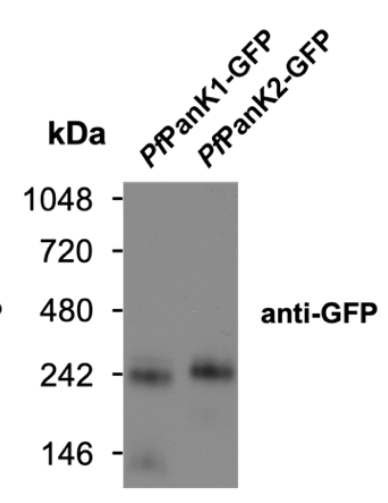

C

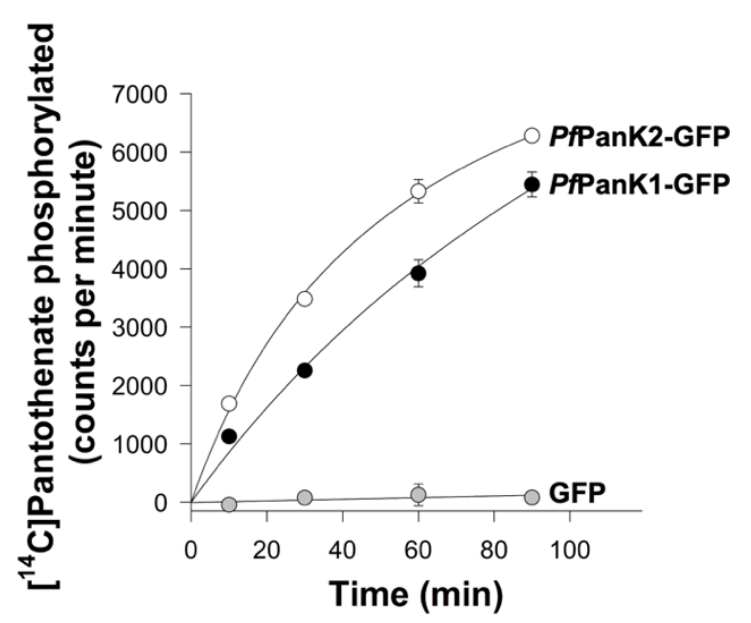

Figure 1. PfPanK1 and PfPanK2 are part of similar-sized protein complexes that possess PanK activity.

(a) Confocal micrographs showing the subcellular location of PfPanK2-GFP within trophozoite/schizont-stage $P$. falciparum-infected erythrocytes. The nuclei of the parasites are labelled with DAPI. From left to right: Brightfield, GFP-fluorescence, DAPI-fluorescence, and merged images. Arrows indicate the plasma membranes of the erythrocyte (red) or the parasite (white). Scale bars represent $5 \mu \mathrm{m}$. (b) Denaturing and native western blot analyses of the GFP-tagged proteins in PfPanK1-GFP and PfPanK2-GFP expressing parasites. The expected sizes of the proteins are $\sim 87 \mathrm{kDa}$ for PfPanK1-GFP and $\sim 118 \mathrm{kDa}$ for PfPanK2-GFP. The molecular mass of the GFP tag is $\sim 27 \mathrm{kDa}$. Western blots were performed with anti-GFP antibodies and each of the blots shown is representative of three independent experiments, each performed with a different batch of parasites. (c) The phosphorylation of $\left[{ }^{14} \mathrm{C}\right]$ pantothenate (initial concentration of $2 \mu \mathrm{M}, \sim 10,000$ counts per minute) over time by the immunopurified complex from lysates of parasites expressing PfPanK1-GFP (black circles), PfPanK2-GFP (white circles) and untagged GFP (grey circles). Data shown are representative of two independent experiments, each performed with a different batch of parasites and carried out in duplicate.

Error bars represent range/2 and are not shown if smaller than the symbols.

To elucidate the protein composition of the PfPanK1-GFP and PfPanK2-GFP complexes, the immunoprecipitated samples were subjected to mass spectrometry (MS)-based proteomic analysis (bound fractions of untagged GFP-expressing and 3D7 wild-type 
parasites were included as negative controls). Both PfPanK1 (36 - 50\% coverage, Figure

129 S2) and PfPanK2 (29 - 49\% coverage, Figure S3) were unequivocally detected as the two

130 most abundant proteins in the immunopurified complex from both the PfPanK1-GFP- and

131 PfPanK2-GFP-expressing cells (Figure 2a). Interestingly the next most abundant protein

132 detected in both complexes was Pf14-3-3I (43 - 67\% coverage, Figure 2a and Figure S4).

133 These results are consistent with PfPanK1, PfPanK2 and Pf14-3-3I being part of the same

134 protein complex. Other proteins, such as M17 leucyl aminopeptidase (fourth most abundant),

135 were also detected in the MS analysis, albeit with a comparatively fewer number of peptides

136 (Figure 2a, and Table S3).

138 To test further whether PfPanK1 and PfPanK2 are part of the same protein complex, we 139 introduced episomally-expressed PfPanK2-GFP into parasite strains generated in a 140 previous study ${ }^{27}$. These mutant strains, termed $\mathrm{PanOH}-\mathrm{A}, \mathrm{PanOH}-\mathrm{B}$ and CJ-A, were 141 generated by drug-pressuring parasites with antiplasmodial pantothenate analogues, and 142 harbour mutations in PfPanK1 that affect PfPanK catalytic activity ${ }^{27}$. We immunopurified 143 the PfPanK2-GFP complex from the PanOH-A, PanOH-B and CJ-A strains, as well as from 144 wild type (Parent) parasites that expresses PfPanK2-GFP as a control, and performed $145\left[{ }^{14} \mathrm{C}\right]$ pantothenate phosphorylation assays on the immunopurified PfPanK2-GFP complex. 146 As we reported previously, the PfPanK1 mutations alter the PfPanK activity of PanOH-A, 147 PanOH-B and CJ-A parasites such that the following rank order of enzyme activity relative 148 to the Parent line is observed: $\mathrm{PanOH}-\mathrm{A}>\mathrm{Parent}>\mathrm{PanOH}-\mathrm{B}>\mathrm{CJ}-\mathrm{A}$ (Figure $\mathbf{2 b}(\mathbf{i}),{ }^{27}$ ). 149 Notably, PanK activity of the immunopurified PfPanK2-GFP complex from the various 150 PfPanK2-GFP expressing lines followed the same rank order (i.e. PanOH-A ${ }^{+P f P a n k 2-G F P}>$ 151 Parent $\left.^{+ \text {PPPanK2-GFP }}>\mathrm{PanOH}-\mathrm{B}^{+ \text {PPPanK2-GFP }}>\mathrm{CJ}^{+\mathrm{A}^{+P F P a n K 2-G F P}}\right)$. This difference in 152 pantothenate phosphorylation rates was not due to variations in the amount of PfPanK2153 GFP protein in the immunopurified complexes used for the assays (Figure S5). These data 154 are consistent with PfPanK2-GFP associating with the mutant PfPanK1 from each cell line 155 and indicate that both proteins are part of the same PanK complex in Plasmodium parasites.

157 Our proteomic analysis identified Pf14-3-3I as being co-immunoprecipitated with both 158 PfPanK1 and PfPanK2 (Figure 2a). To test whether Pf14-3-3I is a bona fide component of 159 the PanK complex of $P$. falciparum, we performed western blotting with a pan-specific anti160 14-3-3 antibody. Under native conditions, the 14-3-3 antibody detected a major protein band 161 at $<66 \mathrm{kDa}$, which likely represents dimeric Pf14-3-3I proteins from the parasite ${ }^{31}$. We also 162 observed a protein complex of $\sim 240 \mathrm{kDa}$ in each of the PfPanK1-GFP-, PfPanK2-GFP- and 
163 untagged GFP-expressing parasites (solid arrow, Figure 2c). In addition, a protein complex 164 of slightly higher molecular mass, likely corresponding to the PanK complex that includes 165 the GFP epitope tag, was also observed in the PfPanK1-GFP-, PfPanK2-GFP-expressing 166 parasites but not the untagged GFP-expressing parasites (dashed arrow, Figure 2c). As a 167 direct test for whether Pf14-3-3I exists in the same complex as PfPanK1 and PfPanK2, we 168 performed western blotting on proteins immunopurified with anti-GFP antibodies from the 169 PfPanK1-GFP-, PfPanK2-GFP- and untagged GFP-expressing parasites. We found that 170 Pf14-3-3I protein was detected in the immunopurified complex from both the PfPanK1-GFP171 and PfPanK2-GFP-expressing cells, but not in that purified from untagged GFP-expressing 172 cells (Figure 2c). Together with the native western blot (Figure 1b) and proteomic (Figure 173 2a) analyses, these results are consistent with PfPanK1 and PfPanK2 being part of the same 174 complex that also contains Pf14-3-3I, and that this complex is responsible for the PanK 175 activity observed in the intraerythrocytic stage of $P$. falciparum. 


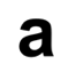

\begin{tabular}{|c|c|c|c|c|}
\hline \multirow[b]{3}{*}{ Protein detected } & \multicolumn{4}{|c|}{ No of peptides detected ( $>95 \%$ confidence) } \\
\hline & \multicolumn{2}{|c|}{$\begin{array}{c}\text { PfPanK1-GFP line } \\
\text { co-immunoprecipitation }\end{array}$} & \multicolumn{2}{|c|}{$\begin{array}{c}\text { PfPanK2-GFP line } \\
\text { co-immunoprecipitation }\end{array}$} \\
\hline & $1^{\text {st }}$ rep & $2^{\text {nd }}$ rep & $1^{\text {st }}$ rep & $2^{\text {nd }}$ rep \\
\hline PfPanK2 & 10 & 24 & 16 & 77 \\
\hline PfPanK1 & 20 & 23 & 19 & 49 \\
\hline 14-3-3 protein $(P f 14-3-3 I)$ & 7 & 14 & 16 & 41 \\
\hline M17 leucyl aminopeptidase & 3 & 4 & 5 & 8 \\
\hline
\end{tabular}

\section{b}

(i)

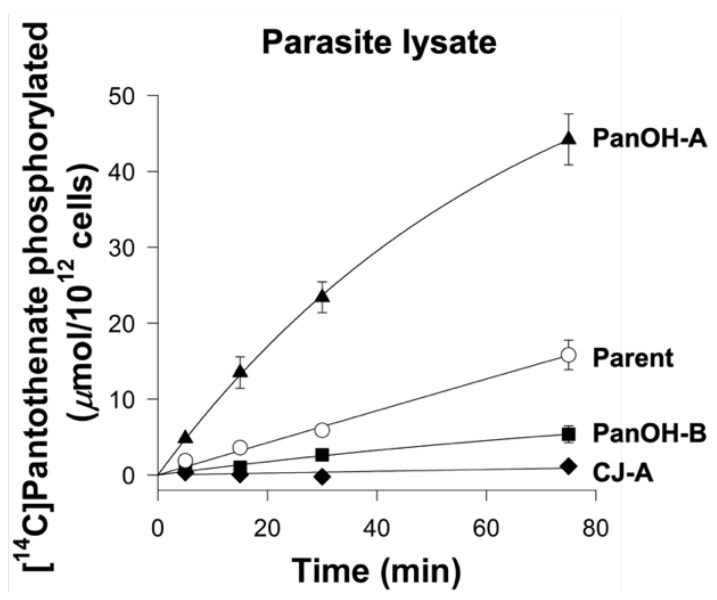

C

Native

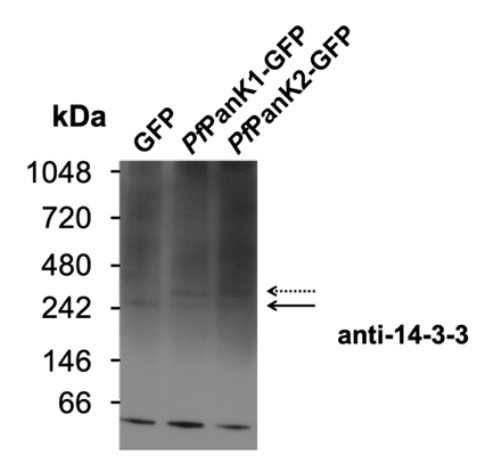

(ii)

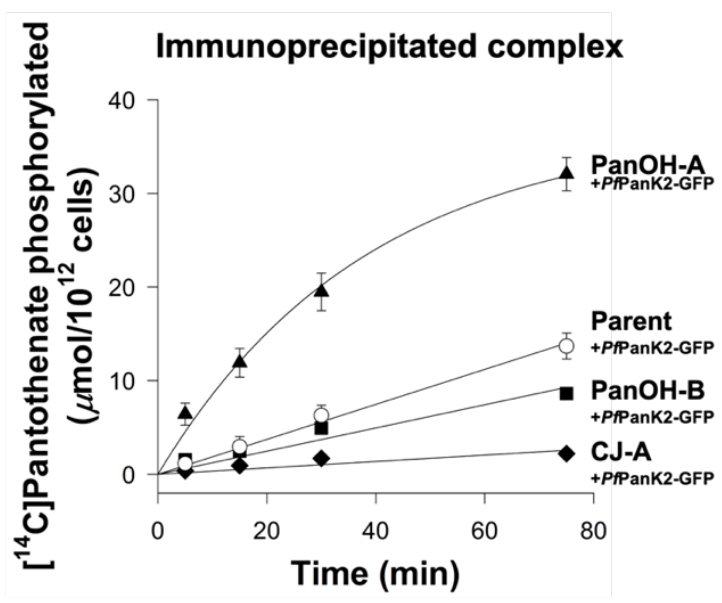

Figure 2. PfPanK1 and PfPanK2 are part of a single PanK complex that includes Pf14-3-3I. (a) The four most abundant proteins identified in the MS analysis of proteins immunoprecipitated with anti-GFP antibodies from PfPanK1-GFP- and PfPanK2-GFP-expressing parasites. Data shown are representative of two independent analyses ( $1^{\text {st }}$ and $2^{\text {nd }}$ rep), each performed with a different batch of parasites. Only proteins with three or more peptides detected in both replicate co-immunoprecipitation experiments are shown. Proteins detected in the untagged GFP-expressing or wild-type 3D7 parasite immunoprecipitations (negative controls) were removed. Proteins are listed in descending order according to the total number of peptides detected 
across all replicates (all four column). (b) The phosphorylation of $\left[{ }^{14} \mathrm{C}\right]$ pantothenate (initial concentration of 2 $\mu \mathrm{M}$ ) over time by (i) lysates generated from Parent (white circles), PanOH-A (black triangles), $\mathrm{PanOH}-\mathrm{B}$ (black squares) and CJ-A (black diamonds) parasites (reproduced from ${ }^{27}$ ) and (ii) proteins immunoprecipitated with anti-GFP antibodies from Parent ${ }^{+P \text { PPank2-GFP }}$ (white circles), PanOH-A ${ }^{+P \text { PPank2-GFP }}$ (black triangles), $\mathrm{PanOH}$ $\mathrm{B}^{\text {+PPPank2-GFP }}$ (black squares) and CJ-A ${ }^{\text {+PPPank2-GFP }}$ (black diamonds) parasite lysates. Values in (ii) are averaged from three independent experiments, each performed with a different batch of parasites and carried out in duplicate. Error bars represent SEM and are not shown if smaller than the symbols. (c) Native western blot analysis of the lysates and denaturing western blot analyses of the different GFP-trap co-immunoprecipitation fractions of PfPanK1-GFP- and PfPanK2-GFP-expressing parasites, with untagged GFP-expressing parasite as a control. Western blots were performed with pan-specific anti-14-3-3 antibodies (previously shown to detect Plasmodium 14-3-3 ${ }^{32}$ ). Arrows indicate the position of 14-3-3-containing complexes of comparable masses to the complexes found in PfPanK1-GFP- and PfPanK2-GFP-expressing parasites. The native blot shown is a representative of three independent experiments, while the denaturing blot is a representative of two independent experiments, each performed with a different batch of parasites.

\section{TgPanK1 and TgPanK2 also constitute a single complex with PanK activity that is} essential for parasite proliferation

Based on sequence similarity, TgPanK1 and TgPanK2 are homologous to their $P$. falciparum counterparts (Figure S6). To begin to characterise TgPanK1 and TgPanK2, we introduced the coding sequence for a mini-Auxin-Inducible Degron (mAID)-haemagglutinin (HA) tag into the 3' region of the open reading frames of TgPanK1 or TgPanK2 in RH $\triangle K u 80: T I R 1$ strain T. gondii parasites ${ }^{33}$ also expressing a 'tdTomato' red fluorescent protein (RFP). Gene models with inserts sizes are noted (Figure S7a), and successful integration

211 TgPanK1-mAIDHA and TgPanK2-mAIDHA proteins have molecular masses of $\sim 160$ and $212 \sim 200 \mathrm{kDa}$, respectively (Figure 3a), corresponding to the predicted sizes of $\operatorname{TgPanK1}$ 213 mAIDHA (141 kDa) and TgPanK2-mAIDHA (187 kDa). When analysed under native 214 conditions, TgPanK1-mAIDHA and TgPanK2-mAIDHA both exist in protein complexes of $\sim 720 \mathrm{kDa}$ in mass (Figure 3a).

To investigate if $\mathrm{TgPanK} 1$ and TgPanK2 are part of the same $\sim 720 \mathrm{kDa}$ complex, we 218 introduced a sequence encoding a GFP tag into the genomic locus of TgPanK1 in the 219 TgPanK2-mAIDHA strain (integration verified by PCR (Figure S7a and S7c)). Co220 immunoprecipitation experiments revealed that TgPanK1-GFP co-purified with TgPanK2221 mAIDHA (Figure 3b). Analogous experiments with a TgPanK1-HA/TgPanK2-GFP line, wherein we integrated a sequence encoding a GFP tag into the TgPanK2 locus and a sequence encoding a HA tag into the TgPanK1 locus (integration verified by PCR (Figure 
224 S7a and S7d)), yielded similar results (Figure S8). We therefore conclude that, like PfPanK1 225 and PfPanK2 in P. falciparum (Figures 1 and 2), TgPanK1 and TgPanK2 are components 226 of the same protein complex.

228 To determine whether the TgPanK1/TgPanK2 complex has pantothenate kinase activity, we 229 immunopurified proteins from TgPanK1-GFP/TgPanK2-mAIDHA, TgPanK1-HA/TgPanK2230 GFP and control (expressing untagged GFP) cell lines using GFP-Trap, and measured the 231 ability of the purified proteins to phosphorylate pantothenate. The samples purified from the 232 TgPanK1-GFP/TgPanK2-mAIDHA and TgPanK1-HA/TgPanK2-GFP lines exhibited higher 233 pantothenate phosphorylation activity than that from the control untagged GFP-expressing 234 line (Figure 3c). These findings indicate that, like the $P$. falciparum PanKs (Figure 1 and 235 2), the TgPanK1/ TgPanK2 complex possesses PanK activity. 
a
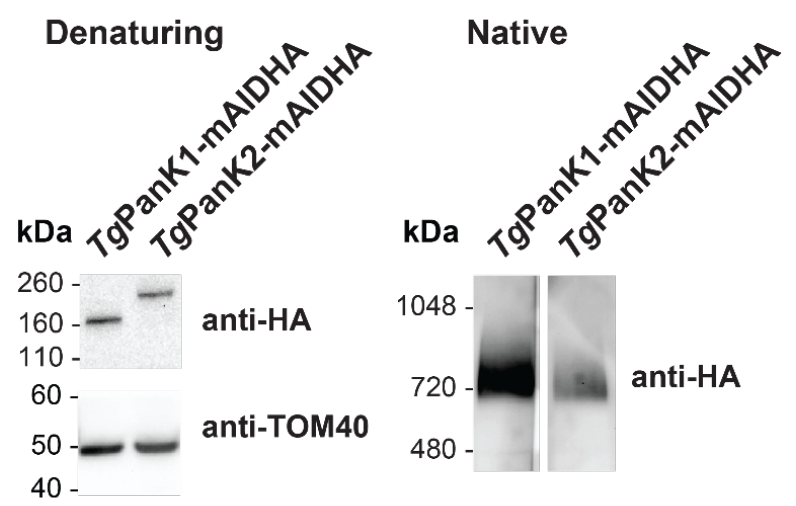

b
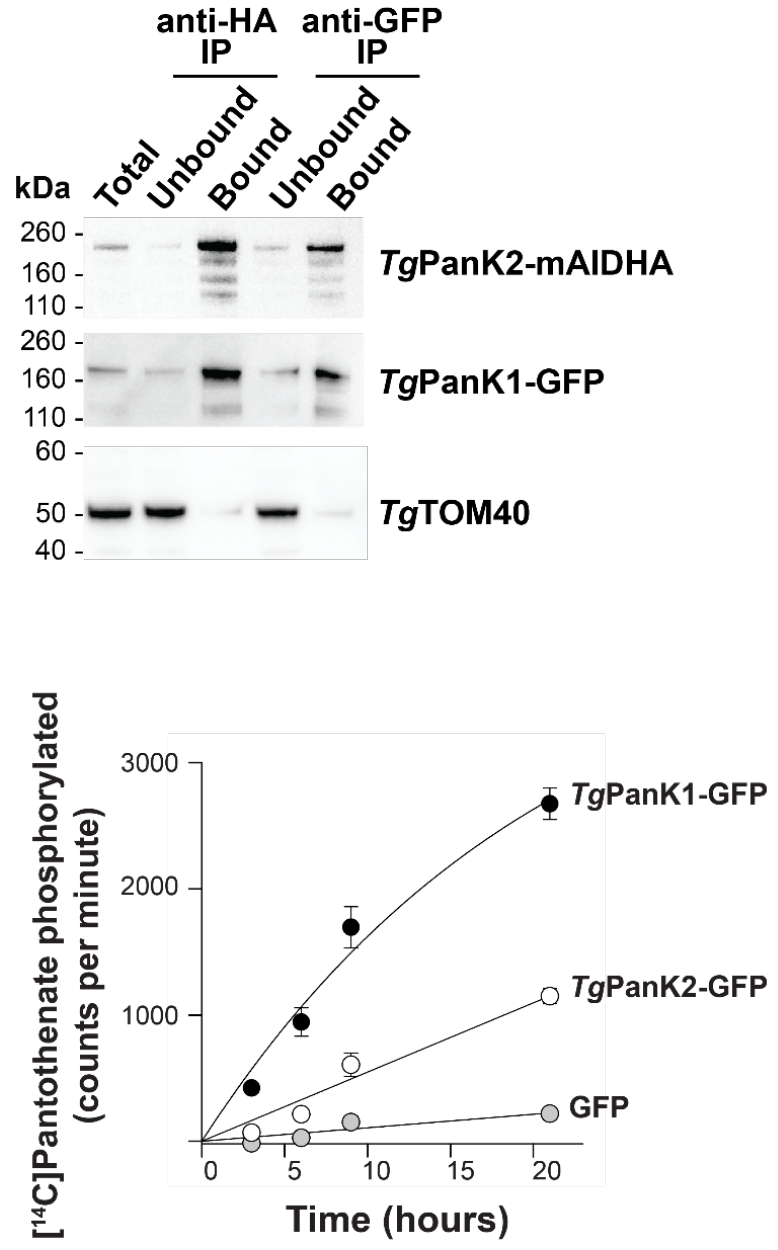

Figure 3. TgPanK1 and TgPanK2 are part of a single protein complex with PanK activity. (a) Denaturing and native western blot analyses of the HA-tagged proteins in TgPanK1-mAIDHA and TgPanK2-mAIDHA expressing parasites. The expected sizes of TgPanK1-mAIDHA and TgPanK2-mAIDHA are $\sim 141 \mathrm{kDa}$ and $\sim 187 \mathrm{kDa}$, respectively. Western blots were performed with an anti-HA antibody and each blot is representative of three independent experiments, each performed with different batches of parasites. Denaturing western blots were also probed with anti-TgTOM40, which served as a loading control. (b) Western blot analysis of proteins from TgPanK1-GFP/TgPanK2-mAIDHA parasite lysates immunoprecipitated with GFP-Trap and antiHA beads. Protein samples were collected before immunoprecipitation (Total), from the fraction not bound to the GFP-Trap nor anti-HA beads (Unbound), and from the fraction bound to the GFP-Trap/anti-HA beads 
(Bound). Membranes were probed with anti-GFP and anti-HA antibodies, and the blot shown is representative of three independent experiments, each performed with different batches of parasites. TgTOM40 served as a control protein that is part of an unrelated protein complex. Bound fractions contain protein from $4 \times$ as many cells as the total and unbound lanes. (c) The phosphorylation of $\left[{ }^{14} \mathrm{C}\right]$ pantothenate (initial concentration $2 \mu \mathrm{M}$ ) over time by protein samples immunoprecipitated with GFP-Trap from parasites expressing TgPanK1GFP/TgPanK2-mAIDHA (black circles),TgPanK1-HA/TgPanK2-GFP (white circles) and untagged GFP (grey circles). Data shown are representative of two independent experiments, each performed with a different batch of parasites and carried out in duplicate. Error bars represent range/2 and are not shown if smaller than the symbols.

As is the case for PfPanK2, the nucleotide-binding motifs of TgPanK2 deviate substantially from those of other eukaryotic PanKs (Figure S6). It is therefore unclear whether pantothenate phosphorylation is catalysed solely by TgPanK1 or if TgPanK2 also contributes to PanK activity. To answer this, we first investigated whether TgPanK1 and TgPanK2 are important for parasite proliferation. TgPanK1 and TgPanK2 were individually knocked down by exposing the mAID-regulated lines to $100 \mu \mathrm{M}$ indole-3-acetic acid (IAA a plant hormone of the auxin class), a concentration that we determined was not detrimental 264 to wild-type parasite proliferation. TgPanK1-mAIDHA and TgPanK2-mAIDHA were degraded within an hour of exposing the parasites to IAA (Figure 4a). Both the TgPanK1mAIDHA and TgPanK2-mAIDHA lines express RFP, which enabled us to monitor parasite proliferation using fluorescence growth assays, as described previously ${ }^{34}$. We measured proliferation of the TgPanK1-mAIDHA, TgPanK2-mAIDHA and parental lines cultured in the presence or absence of $100 \mu \mathrm{M}$ IAA over seven days. In the absence of IAA, we observed a normal sigmoidal growth curve for all three strains (Figure 4b). By contrast, we observed a complete cessation of proliferation of both the TgPanK1-mAIDHA- and TgPanK2mAIDHA-expressing parasite lines, but not the parental strain, in the presence of $100 \mu \mathrm{M}$ IAA (Figure 4b). These data indicate that both TgPanK1 and TgPanK2 are crucial for $T$. gondii proliferation and, notably, that neither can substitute for the other. To establish whether TgPanK1 and TgPanK2 are essential due to the PanK activity of the complex, we constitutively expressed the type II PanK of Staphylococcus aureus (Sapank) in both the TgPanK1-mAIDHA- and TgPanK2-mAIDHA-expressing parasite lines, generating lines that we termed TgPanK1-mAIDHA ${ }^{+S a P a n K-T y 1}$ and TgPanK2-mAIDHA ${ }^{+S a P a n K-T y 1}$. The expression of the SaPanK protein in these strains was verified by immunofluorescence microscopy and western blot (Figure S9). We measured the proliferation of the TgPanK1-mAIDHA ${ }^{+S a P a n K-T y 1}$

281 and TgPanK2-mAIDHA ${ }^{+ \text {SaPanK-Ty1 }}$ lines in the presence and absence of $100 \mu \mathrm{M}$ IAA, and compared this with expression of the TgPanK1-mAIDHA, TgPanK2-mAIDHA and parental 
283 lines. We obtained fluorescence measurements over a 7 day period, and compared the 284 proliferation of each strain when the parental strain cultured in the absence of IAA reached 285 mid-log phase. We found that both the TgPanK1-mAIDHA+SaPanK-Ty1 and TgPanK2$286 \mathrm{mAIDHA}^{+S a P a n k-T y 1}$ lines proliferated at a similar rate to the parental control line when 287 cultured in the presence of IAA, in contrast to the TgPanK1-mAIDHA and TgPanK2-mAIDHA 288 lines, where minimal proliferation was observed (Figure 4c). Collectively, our studies on 289 TgPanK1 and TgPanK2 reveal that (i) TgPanK1 and TgPanK2 are part of the same protein 290 complex, (ii) expression of both is required for PanK activity, and (iii) PanK activity of the 291 complex is important for T. gondii proliferation during the disease-causing tachyzoite stage. 
a

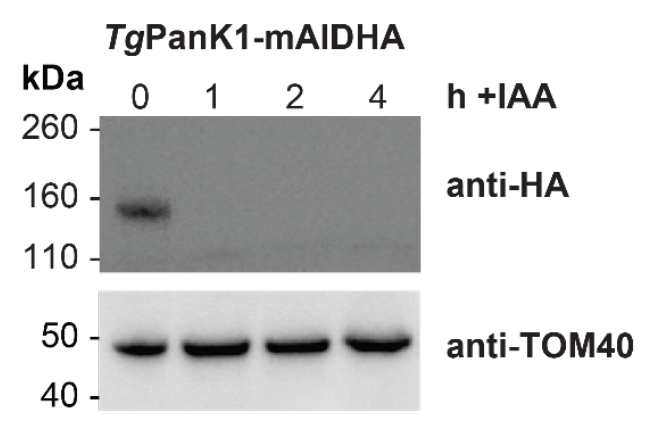

TgPanK2-mAIDHA
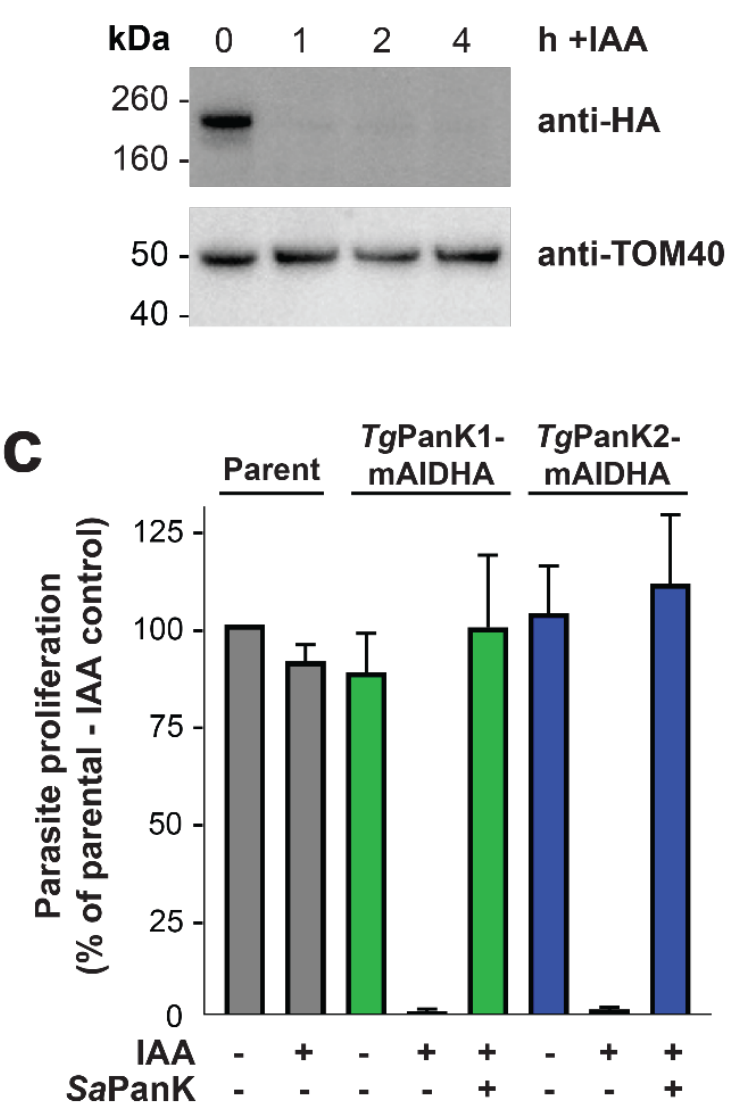

b

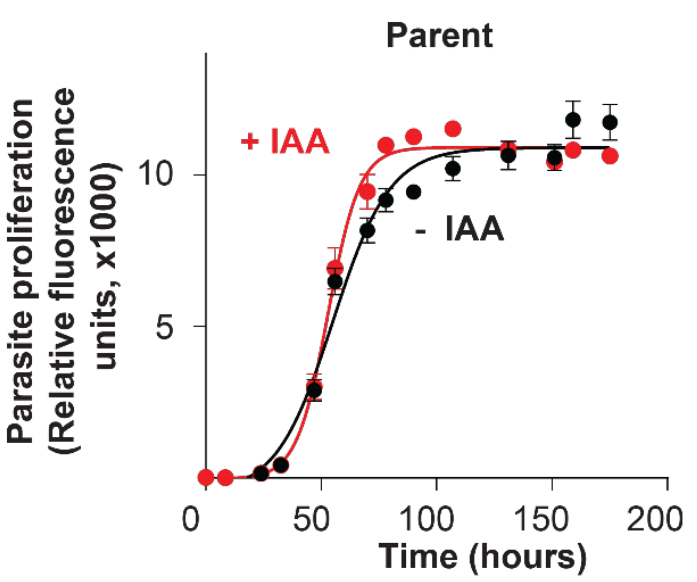

TgPanK1-mAIDHA
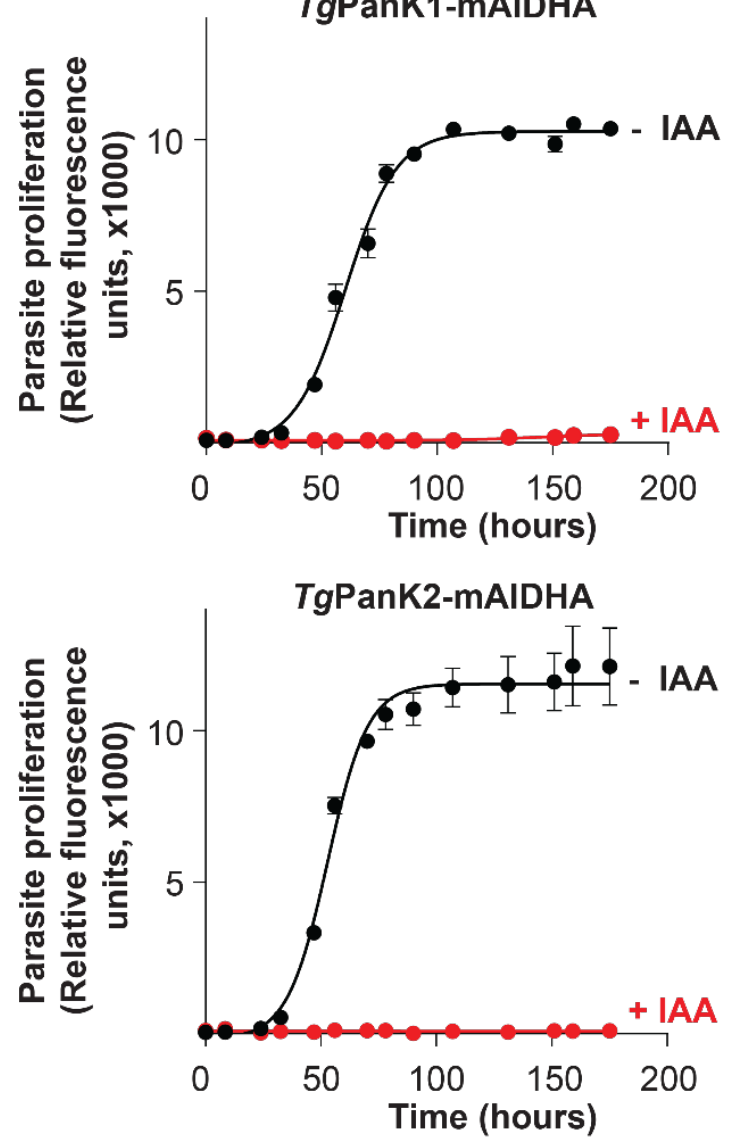

Figure 4. Expression of both TgPanK1 and TgPanK2 is necessary for PanK activity and for T. gondii tachyzoite proliferation. (a) IAA-induced knockdown of TgPanK1-mAIDHA or TgPanK2-mAIDHA protein over time. Western blot analysis of TgPanK1-mAIDHA- and TgPanK2-mAIDHA-expressing parasites, either in the absence of, or after 1, 2 and 4 hours of exposure to $100 \mu \mathrm{M} \mathrm{IAA}$. Membranes were probed with antiHA antibody to detect the TgPanK1-mAIDHA and TgPanK2-mAIDHA proteins, and anti-TgTom40 as a loading control. Western blots are representative of three independent experiments, each performed with a different batch of parasites. (b) The effect of TgPanK1-mAIDHA or TgPanK2-mAIDHA knockdown on $T$. gondii tachyzoite proliferation. tdTomato RFP-expressing parasites (Parent, TgPanK1-mAIDHA and TgPanK2-mAIDHA) were cultured over 7 days in the presence (red circles) or absence (black circles) of 100 IM IAA. Parasite proliferation was measured over time by assessing the RFP expression using a fluorescence reader. Graphs shown are representative of three independent experiments carried out in 
triplicate, each performed with a different batch of parasites. Error bars represent SD and are not shown if smaller than the symbols. (c) Complementation of TgPanK1 and TgPanK2 knockdown with S. aureus PanK. S. aureus PanK was constitutively expressed in TgPanK1-mAIDHA (green bars) and TgPanK2-mAIDHA (blue bars) parasites. The Parent RH RFP (grey bars), non-complemented and SaPanK-Ty1-complemented parasites were cultured alongside the TgPanK1-mAIDHA and TgPanK2-mAIDHA lines in the presence (+) or absence (-) of $100 \mu \mathrm{M}$ IAA. Parasite proliferation was monitored 1-2 times daily for 7 days. Proliferation was compared when the parental strain cultured in the absence of IAA was at the mid-log phase of parasite proliferation. Values are averaged from three independent experiments, each performed with a different batch of parasites and carried out in triplicate. Error bars represent SEM.

\section{DISCUSSION}

All PanKs characterised to date have been shown to exist as homodimers ${ }^{3-10}$. Here we present data consistent with PanK1 and PanK2 of the apicomplexan parasites $P$. falciparum and T. gondii forming a heteromeric complex (Figure 2 and Figure 3), a hitherto undescribed phenomenon in nature.

There have been several unsuccessful attempts by us [unpublished] and others ${ }^{35-37}$ to express a functional PfPanK1. Whilst the protein has been successfully expressed in soluble form using various heterologous expression systems ( $E$. coli, insect cells, S. cerevisiae), no study has reported PanK activity from the heterologously-expressed and purified protein. Our observation here that the presence of PfPanK2 (as well as, potentially, additional proteins) is inextricably linked to PanK activity probably explains why these previous attempts of expressing an active PfPanK1 were unsuccessful ${ }^{35-37 .}$

A comparison of the amino acid sequences of $P$. falciparum and $T$. gondii PanKs with those of other type II PanKs, such as human PanK3 (HsPanK3), provides a possible explanation for why PanKs from these apicomplexan parasites exist in heteromeric complexes (Figure S6). Each of the two identical active sites of the homodimeric HsPanK3 are formed by parts of both of its protomers. Certain residues form hydrogen bonds with pantothenate (Glu138, Ser195, Arg207 from one protomer and Val268' and Ala269' from the second protomer), while others interact to stabilise the active site (Asp137 with Tyr258', and Glu138 with Tyr254') ${ }^{38,39}$ (Figure S10). Notably, the hydrogen bond between Glu138 and Tyr254' is important for the allosteric activation of the enzyme ${ }^{39}$. Critically, one of the important residues involved in active site stabilisation, Asp137, is only conserved in the PanK1 of $P$. falciparum and T. gondii but not their PanK2, while others, such as Tyr254' and Tyr258' are conserved in their PanK2 but not PanK1 (Figure $\mathbf{S 6}$ and S10). This raises the possibility 
341 that PanK1 and PanK2 homodimers are likely not functional, and that only a heteromeric

342 PanK1/PanK2 complex, with a single complete active site, can serve as a functional PanK 343 enzyme in these apicomplexan parasites. This is consistent with the previous observation 344 that two of the nucleotide-binding motifs of PfPanK2 deviate from those of other eukaryotic 345 PanKs 28. Whether the incomplete second active site plays an additional, as yet 346 undetermined, role(s) remains to be seen. It should be noted that the PanKs of other 347 apicomplexan parasites exhibit a similar conservation of residues as that described above 348 for $P$. falciparum and T. gondii (Figure S11), raising the possibility that heteromeric PanK complexes are ubiquitous in Apicomplexa.

The apparent molecular weight of the PfPanK heterodimer complex (as determined from native western blotting) is consistent with that of a complex that includes PfPanK1, PfPanK2 and a Pf14-3-3I dimer (Figure 1b). However, due to various limitations of native gels ${ }^{40}$, it is difficult to obtain an accurate estimate of the molecular weight of the complex. Although we cannot completely rule out the inclusion of other proteins in the PfPanK complex, such as M17 leucyl aminopeptidase (Figure 2a and Table S3), we think that this is unlikely, since peptides from these proteins were detected at lower abundance than peptide from PfPanK1, PfPanK2 and Pf14-3-3I. The role of Pf14-3-3I in the heteromeric PfPanK complex (Figure $\mathbf{2 a}$ and $\mathbf{2 c}$ ) is not clear. The 14-3-3 protein family comprises highly conserved proteins that 360 occur in a wide array of eukaryotic organisms, including apicomplexans such as $P$. 361 falciparum 32,41-43. Multiple isoforms of 14-3-3 are found to occur in every organism that 362 expresses the protein ${ }^{44}$. 14-3-3 proteins bind to, and regulate, the function of proteins that 363 are involved in a large range of cellular functions, including cell cycle regulation, signal 364 transduction and apoptosis (reviewed in ${ }^{45}$ ). They typically bind to phosphorylated Ser/Thr residues on target proteins, and modify their target protein's trafficking/targeting (reviewed in ${ }^{46}$ ), conformation, co-localisation, and/or activity (reviewed in ${ }^{47}$ ). We speculate that Pf14-

367 3-3I plays a regulatory role in the PfPanK complex. The TgPanK heterodimer complex has 368 a molecular weight that is much larger than the combined molecular weights of $\operatorname{TgPanK} 1$ 369 and TgPanK2. Unfortunately, mass spectrometry analysis of the T. gondii PanK complex was unsuccessful, presumably because the native level of expression of the complex is too low.

373 In this study, we have characterised, for the first time, PanK activity in T. gondii. The $374\left[{ }^{14} \mathrm{C}\right]$ pantothenate phosphorylation data generated with the purified TgPanK complex 375 (Figure 3c) provide the first biochemical evidence indicating that these putative PanKs are 
able to phosphorylate pantothenate. This finding, combined with the results of the knockdown and SaPanK complementation experiment in T. gondii (Figure 4b and 4c), not only demonstrate the essentiality of TgPanK1 and TgPanK2, but also show that the essentiality is due to their role in phosphorylating pantothenate.

T. gondii parasites inhabit metabolically active mammalian cells that contain their own CoA biosynthesis pathway. Our data indicate that $T$. gondii parasites are unable to scavenge sufficient downstream intermediates in the CoA biosynthesis pathway from their host cells, including CoA, for their survival, and therefore must maintain their own active CoA biosynthesis pathway. The requirement for CoA biosynthesis in $T$. gondii, coupled with the intense investigation of this pathway as a drug target in $P$. falciparum ${ }^{27,37,48-61}$, suggests that further characterisation of TgPanK, and the CoA biosynthesis pathway in T. gondii, could yield novel drug targets for chemotherapy.

It has been an open question as to why many organisms (eukaryotes ${ }^{11-21}$ and prokaryotes 7,23-25), including all apicomplexan parasites ${ }^{62}$, express more than one PanK and why some PanKs appear to be non-functional 7,21,23 (either by analysis of their sequence or through failed attempts to demonstrate PanK activity experimentally). The data that we present here provides a possible answer to this question.

\section{METHODS}

\section{Parasite and host cell culture}

P. falciparum parasites were maintained in RPMI 1640 media supplemented with $11 \mathrm{mM}$ glucose (to a final concentration of $22 \mathrm{mM}$ ), $200 \mu \mathrm{M}$ hypoxanthine, $24 \mu \mathrm{g} / \mathrm{mL}$ gentamicin and $6 \mathrm{~g} / \mathrm{L}$ Albumax II as described previously ${ }^{63}$. T. gondii was cultured in human foreskin fibroblasts (HFF cells) as described previously ${ }^{64}$. T. gondii parasites were grown in flasks with a confluent HFF cell layer in either Dulbecco's modified Eagle's medium (DMEM) or complete RPMI 1640, with both media containing $2 \mathrm{~g} / \mathrm{L}$ sodium bicarbonate and supplemented with $1 \%(\mathrm{v} / \mathrm{v})$ fetal bovine serum (FBS), 50 units $/ \mathrm{mL}$ penicillin, $50 \mu \mathrm{g} / \mathrm{mL}$ streptomycin, $10 \mu \mathrm{g} / \mathrm{mL}$ gentamicin, $0.2 \mathrm{mM} \mathrm{L-glutamine,} \mathrm{and} 0.25 \mu \mathrm{g} / \mathrm{mL}$ amphotericin B.

\section{Plasmid preparation and parasite transfection}

The PfPanK1-GFP-expressing cell line was generated in a previous study ${ }^{27}$, while the untagged GFP line was a generous gift from Professor Alex Maier (Research School of 
410 Biology, Australian National University, Canberra). A Pfpank2-pGlux-1 vector was

411 generated for the overexpression of PfPanK2-GFP in 3D7 strain $P$. falciparum as detailed

412 in the SI. The primers used are listed in Table S1. The same construct was also transfected 413 into each of the mutant clones and their Parent line described previously by Tjhin et al. ${ }^{27}$. 414 Transfections were performed with ring-stage parasites and transformants were 415 subsequently selected and maintained using WR99210 (10 nM) as described previously ${ }^{65}$.

417 The TgPanK1-mAIDHA and TgPanK2-mAIDHA expressing lines were generated using a 418 CRISPR/Cas9 strategy as previously described in Shen et al. ${ }^{66}$, which is detailed in the SI. 419 The guide RNAs, primers, and the sequences of gBlocks used are provided in Table S1 420 and S2.

422 The complementation lines TgPanK1-mAIDHA ${ }^{+S a P a n K-T y 1}$ and TgPanK2-mAIDHA ${ }^{+S a P a n K-T y 1}$ 423 were created by expressing the $S$. aureus type II PanK gene (Sapank) in T. gondii under the 424 regulation of the tubulin promoter (details in the SI and Table S1 and S2).

\section{Immunofluorescence assays and microscopy}

427 Fixed PfPanK2-GFP-expressing 3D7 strain $P$. falciparum parasites within infected 428 erythrocytes were observed and imaged with a Leica TCS-SP2-UV confocal microscope 429 (Leica Microsystems) using a $63 \times$ water immersion lens as described in the SI. To confirm 430 the expression of SaPanK-Ty1 in the TgPanK1-mAIDHA ${ }^{+S a P a n K-T y 1}$ line, 431 immunofluorescence assays were performed based on the protocol described by van 432 Dooren et al. ${ }^{67}$. T. gondii parasites were incubated with mouse anti-Ty1 antibodies (1:200 433 dilution). Secondary antibodies used were goat anti-mouse AlexaFluor 488 at a 1:250 434 dilution. The nucleus was stained with DAPI. Immunofluorescence images were acquired 435 on a DeltaVision Elite system (GE Healthcare) using an inverted Olympus IX71 microscope 436 with a $100 \times$ UPlanSApo oil immersion lens (Olympus) paired with a Photometrics 437 CoolSNAP $\mathrm{HQ}^{2}$ camera. Images taken on the DeltaVision setup were deconvolved using 438 SoftWoRx Suite 2.0 software. Images were adjusted linearly for contrast and brightness.

440 Polyacrylamide gel electrophoresis and western blotting

441 Parasite samples were analysed using either denaturing or blue native gels to determine 442 the presence and abundance of a single protein or protein complex of interest, respectively. 443 Briefly, mature trophozoite-stage $P$. falciparum parasites were isolated from infected 
erythrocytes by saponin lysis, as described previously ${ }^{68}$. Saponin-isolated parasites were then pelleted and lysed in the appropriate buffers (as detailed in the SI). T. gondii protein samples were prepared as described previously, with samples for blue native-PAGE solubilised in Native PAGE sample buffer (ThermoFisher) containing 1\% (v/v) Triton X-100 67. Protein samples generated from both $P$. falciparum and $T$. gondii parasites were separated by polyacrylamide gel electrophoresis (PAGE) in precast NuPAGE $(4-12 \%$ or $12 \%$ ) or NativePAGE (4-16\%) gels (ThermoFisher) according to the manufacturer's instructions with minor modifications (detailed in the SI). The separated proteins were transferred to the appropriate membranes (nitrocellulose or polyvinylidene fluoride (PVDF)) and blocked (detailed in the SI) before immunoblotting. Blocked membranes were exposed (45 $\min -2 \mathrm{~h}$ ) to specific primary and secondary antibodies to allow for the detection of the protein of interest. To visualise the protein band(s), membranes were incubated in Pierce enhanced chemiluminescence (ECL) Plus Substrate (ThermoFisher) according to the manufacturer's instructions or home-made ECL $(0.04 \% \mathrm{w} / \mathrm{v}$ luminol, $0.007 \% \mathrm{w} / \mathrm{v}$ coumaric acid, $0.01 \% \mathrm{v} / \mathrm{v} \mathrm{H}_{2} \mathrm{O}_{2}, 100 \mathrm{mM}$ Tris, $\mathrm{pH}$ 9.35). Protein bands were then either imaged onto X-ray films and scanned or visualised on a ChemiDoc MP Imaging System (Thermo Scientific).

\section{Flow cytometry}

463 Saponin-isolated mature trophozoites from 3D7 wild-type, Parent ${ }^{+P f P a n K 2-G F P}$, PanOH$A^{+P f P a n K 2-G F P}$, PanOH-B ${ }^{+P f P a n K 2-G F P}$ and CJ-A $^{+P f P a n K 2-G F P}$ cultures were subjected to flow cytometry analysis to determine the proportion of GFP-positive cells. Aliquots of each isolated parasite suspension were diluted in a saline solution $(125 \mathrm{mM} \mathrm{NaCl}, 5 \mathrm{mM} \mathrm{KCl}, 25$ mM HEPES, $20 \mathrm{mM}$ glucose and $1 \mathrm{mM} \mathrm{MgCl}_{2}, \mathrm{pH} 7.1$ ) to a concentration of $\sim 10^{6}-10^{7}$ cells $/ \mathrm{mL}$ in $1.2 \mathrm{~mL}$ Costar polypropylene cluster tubes (Corning) and sampled for flow cytometry analysis (in measurements of 100,000 cells, low sampling speed) with the following settings: forward scatter $=450 \mathrm{~V}$ (log scale), side scatter $=350 \mathrm{~V}$ (log scale) and AlexaFluor $488=600 \mathrm{~V}$ (log scale). The 3D7 wild-type cells were used to establish a gating strategy that defined a threshold below which parasites were deemed to be auto-fluorescent.

473 This strategy was then applied in all analyses to determine the proportion of cells in each cell line that was GFP-positive (i.e. above the defined threshold). 


\section{Immunoprecipitations}

480 In order to immunopurify GFP-tagged or HA-tagged proteins from parasite lysates, 481 immunoprecipitation was performed using either GFP-Trap (high affinity anti-GFP alpaca 482 nanobody bound to agarose beads; Chromotek) or anti-HA beads (Sigma-Aldrich), 483 respectively. $P$. falciparum lysate was prepared from saponin-isolated trophozoites, and $T$. 484 gondii lysate was prepared from tachyzoites, as described previously ( ${ }^{68}$ and ${ }^{67}$, respectively). 485 Immunoprecipitation was then performed (as detailed in the SI). In $P$. falciparum 486 experiments where the amount of immunoprecipitated proteins were to be standardised 487 across cell lines and biological repeats, the number of GFP-positive cells to be used for 488 lysate preparation was calculated by a combination of haemocytometer count and flow 489 cytometry. All immunoprecipitated samples from Parent ${ }^{+P P P a n K 2-G F P}, \mathrm{PanOH}-\mathrm{A}^{+P A P a n k 2-G F P}$, 490 PanOH-B ${ }^{+P f P a n K 2-G F P}$ and CJ-A ${ }^{+P f P a n K 2-G F P}$ cell lines contained protein from $5 \times 10^{7}$ GFP491 positive cells. Each of these samples were subsequently divided into two equal aliquots, one 492 used in the $\left[{ }^{14} \mathrm{C}\right]$ pantothenate phosphorylation assay and the other for denaturing western 493 blot.

495 When an aliquot of the immunoprecipitation sample (beads that have bound proteins from $496 \sim 10^{6}-10^{7}$ GFP-positive cells for $P$. falciparum and $\sim 10^{7}-10^{8}$ cells for $T$. gondii) was 497 required for western blot, the bead suspension was centrifuged $(2,500 \times g$, 2 min), the 498 supernatant removed, and the beads resuspended in $50 \mu \mathrm{L}$ sample buffer containing $2 \times$ 499 NuPAGE lithium dodecyl sulfate (LDS) sample buffer (ThermoFisher) and $2 \times$ NuPAGE 500 sample reducing agent (ThermoFisher). In some experiments, $10 \mu \mathrm{L}$ aliquots of the total 501 and unbound lysate fractions were each mixed with $10 \mu \mathrm{L}$ of the same sample buffer. These 502 samples were then boiled $\left(95^{\circ} \mathrm{C}, 10 \mathrm{~min}\right)$ and $10 \mu \mathrm{L}$ of each was then used in a denaturing 503 western blot as described above.

$\left[{ }^{14} \mathrm{C}\right]$ Pantothenate phosphorylation assays

506 In order to determine the PanK activity of the protein(s) isolated in the 507 GFP-Trap immunoprecipitation assays, the immunopurified complexes were used to 508 perform a $\left[{ }^{14} \mathrm{C}\right]$ pantothenate phosphorylation time course. The bead suspensions containing 509 the immunoprecipitated proteins from $P$. falciparum and T. gondii were centrifuged $(2,500 \times$ $510 \mathrm{~g}, 2 \mathrm{~min}$ ), the supernatant removed, and the beads resuspended in $300 \mu \mathrm{L}$ (for T. gondii) or $511500 \mu \mathrm{L}$ (for $P$. falciparum) of buffer containing $100 \mathrm{mM}$ tris(hydroxymethyl)aminomethane 512 (Tris) $-\mathrm{HCl}\left(\mathrm{pH}\right.$ 7.4), $10 \mathrm{mM} \mathrm{ATP}$ and $10 \mathrm{mM} \mathrm{MgCl}_{2}$ (i.e. all reagents were at twice the final 
513 concentration required for the phosphorylation reaction). Each time course was then initiated

514 by the addition of $300 \mu \mathrm{L}$ (for $T$. gondii) or $500 \mu \mathrm{L}$ (for $P$. falciparum) of $4 \mu \mathrm{M}(0.2 \mu \mathrm{Ci} / \mathrm{mL}$ )

$515 \quad\left[{ }^{14} \mathrm{C}\right]$ pantothenate in water (pre-warmed to $37^{\circ} \mathrm{C}$ ), to the bead suspension. Aliquots of each

516 reaction $(50 \mu \mathrm{L}$ in duplicate) were terminated at pre-determined time points by mixing with

$51750 \mu \mathrm{L} 150 \mathrm{mM}$ barium hydroxide preloaded within the wells of a 96-well, $0.2 \mu \mathrm{m}$ hydrophilic

518 PVDF membrane filter bottom plate (Corning). Phosphorylated compounds in each well

519 were then precipitated by the addition of $50 \mu \mathrm{L} 150 \mathrm{mM}$ zinc sulfate to generate the Somogyi

520 reagent ${ }^{69}$, the wells processed, and the radioactivity in the plate determined as detailed

521 previously ${ }^{70}$. Total radioactivity in each phosphorylation reaction was determined by mixing

$52250 \mu \mathrm{L}$ aliquots of each reaction (in duplicate) thoroughly with $150 \mu \mathrm{L}$ Microscint-40

523 (PerkinElmer) by pipetting the mixture at least 50 times, in the wells of an OptiPlate-96 524 microplate (PerkinElmer) ${ }^{70}$.

\section{Mass spectrometry of immunoprecipitated samples}

527 The identities of the proteins co-immunoprecipitated from lysates of parasites expressing 528 PfPanK1-GFP, PfPanK2-GFP and untagged GFP were determined by mass spectrometry. 529 Aliquots of bead-bound co-immunoprecipitated samples were resuspended in $2 \times$ NuPAGE 530 LDS sample buffer and $2 \times$ NuPAGE sample reducing agent and sent (at ambient 531 temperature, travel time less than $24 \mathrm{~h}$ ) to the Australian Proteomics Analysis Facility 532 (Sydney) for processing and mass spectrometry analysis (as detailed in the SI).

\section{Fluorescent T. gondii proliferation assay}

535 Fluorescent $T$. gondii proliferation assays were performed as previously described ${ }^{34}$. Briefly, 5362000 parasites suspended in complete RPMI were added to the wells of optical bottom black 53796 well plates (ThermoFisher) containing a confluent layer of HFF cells, in the presence or 538 absence of $100 \mu \mathrm{M}$ IAA, in triplicate. Fluorescent measurements (Excitation filter, $540 \mathrm{~nm}$; 539 Emission filter, $590 \mathrm{~nm}$ ) using a FLUOstar OPTIMA Microplate Reader (BMG LABTECH) 540 were taken over 7 days.

\section{Knockdown of mAID protein}

543 Flasks containing a confluent layer of HFF cells were seeded with TgPanK1-mAIDHA, 544 TgPanK2-mAIDHA, TgPanK1-mAIDHA ${ }^{+S a P a n K-T y 1}$ or TgPanK2-mAIDHA+SaPanK-Ty1 T. gondii 545 parasites. While the parasites were still intracellular, $100 \mu \mathrm{M}$ of IAA dissolved in ethanol 546 (final ethanol concentration of $0.1 \%, v / v$ ) was added to induce the knockdown of TgPanK1547 mAIDHA or TgPanK2-mAIDHA, with ethanol $(0.1 \%, \mathrm{v} / \mathrm{v})$ added to another flask as a vehicle 
548 control. Flasks with IAA added were processed at 1, 2 and $4 \mathrm{~h}$ time points, and the control

549 flask was processed at the 4-hour time point. Parasite concentrations were determined using

550 a haemocytometer and $1.5 \times 10^{7}$ parasites were resuspended in LDS sample buffer, and 551 boiled at $95{ }^{\circ} \mathrm{C}$ for 10 minutes. An aliquot from each sample was analysed by western 552 blotting.

\section{Alignment of PanK}

555 PanK homologues from $P$. falciparum and T. gondii, and a selection of other type II PanKs 556 from other eukaryotic organisms and $S$. aureus were aligned using PROMALS3D 71 557 (available at: http://prodata.swmed.edu/promals3d/promals3d.php). The default parameters 558 were selected except for the 'Identity threshold above which fast alignment is applied' 559 parameter, which was changed to " 1 " to allow for a more accurate alignment.

\section{ACKNOWLEDGEMENTS}

Proteomics was undertaken at APAF, the infrastructure provided by the Australian Government through the National Collaborative Research Infrastructure Strategy (NCRIS). We are grateful to the Canberra Branch of the Australian Red Cross Blood Service for the provision of red blood cells, and Professor Alex Maier (ANU) for the untagged GFP. expressing $P$. falciparum parasites. ETT, VMH and CS were supported by Research Training Program scholarships from the Australian Government. CS was also funded by an NHMRC Overseas Biomedical Fellowship (1016357). This work was, in part, supported by a Project Grant (APP1129843) from the National Health and Medical Research Council to KJS and a Discovery Grant (DP150102883) from the Australian Research Council to GvD.

The authors declare that they have no conflict of interest.

\section{REFERENCES}

575 1. Leonardi, R., Zhang, Y.-M., Rock, C. O. \& Jackowski, S. Coenzyme A: back in action. Prog. Lipid Res. 44, 125-153 (2005).

2. Spry, C., Kirk, K. \& Saliba, K. J. Coenzyme A biosynthesis: an antimicrobial drug target. FEMS Microbiol. Rev. 32, 56-106 (2008).

3. Yun, M. et al. Structural basis for the feedback regulation of Escherichia coli pantothenate kinase by coenzyme A. J. Biol. Chem. 275, 28093-28099 (2000). 
581 4. Das, S., Kumar, P., Bhor, V., Surolia, A. \& Vijayan, M. Invariance and variability in bacterial PanK: a study based on the crystal structure of Mycobacterium tuberculosis PanK. Acta Crystallogr. D Biol. Crystallogr. 62, 628-638 (2006).

5. Yang, K. et al. Crystal structure of a type III pantothenate kinase: insight into the mechanism of an essential coenzyme A biosynthetic enzyme universally distributed in bacteria. J. Bacteriol. 188, 5532-5540 (2006).

7. Nicely, N. I. et al. Structure of the type III pantothenate kinase from Bacillus anthracis at $2.0 \AA$ resolution: implications for coenzyme A-dependent redox biology. Biochemistry 46, 3234-3245 (2007).

6. Hong, B. S. et al. Prokaryotic type II and type III pantothenate kinases: The same monomer fold creates dimers with distinct catalytic properties. Structure 14, 1251-

8. Hong, B. S. et al. Crystal structures of human pantothenate kinases. Insights into allosteric regulation and mutations linked to a neurodegeneration disorder. J. Biol. Chem. 282, 27984-27993 (2007).

9. $\mathrm{Li}, \mathrm{B}$. et al. Crystal structures of Klebsiella pneumoniae pantothenate kinase in complex with N-substituted pantothenamides. Proteins 81, 1466-1472 (2013).

10. Franklin, M. C. et al. Structural genomics for drug design against the pathogen Coxiella burnetii. Proteins 83, 2124-2136 (2015).

11. Kupke, T., Hernández-Acosta, P. \& Culiáñez-Macià, F. A. 4'-phosphopantetheine and coenzyme A biosynthesis in plants. J. Biol. Chem. 278, 38229-38237 (2003).

12. Tilton, G. B., Wedemeyer, W. J., Browse, J. \& Ohlrogge, J. Plant coenzyme A biosynthesis: characterization of two pantothenate kinases from Arabidopsis. Plant Mol. Biol. 61, 629-642 (2006).

13. Rock, C. O., Calder, R. B., Karim, M. A. \& Jackowski, S. Pantothenate kinase regulation of the intracellular concentration of coenzyme A. J. Biol. Chem. 275, 13771383 (2000).

14. Rock, C. O., Karim, M. A., Zhang, Y.-M. \& Jackowski, S. The murine pantothenate kinase (Pank1) gene encodes two differentially regulated pantothenate kinase isozymes. Gene 291, 35-43 (2002). pantothenate kinase 3 by coenzyme A and coenzyme A thioesters. J. Biol. Chem. 280, 32594-32601 (2005). 
616 17. Zhou, B. et al. A novel pantothenate kinase gene (PANK2) is defective in Hallervorden-Spatz syndrome. Nat. Genet. 28, 345-349 (2001).

18. $\mathrm{Ni}, \mathrm{X}$. et al. Cloning and characterization of a novel human pantothenate kinase gene. Int. J. Biochem. Cell Biol. 34, 109-115 (2002).

19. Hörtnagel, K., Prokisch, H. \& Meitinger, T. An isoform of hPANK2, deficient in pantothenate kinase-associated neurodegeneration, localizes to mitochondria. Hum. Mol. Genet. 12, 321-327 (2003).

20. Ramaswamy, G., Karim, M. A., Murti, K. G. \& Jackowski, S. PPARa controls the

21. Zhang, Y.-M. et al. Chemical knockout of pantothenate kinase reveals the metabolic

23. Awasthy, D. et al. Essentiality and functional analysis of type I and type III pantothenate kinases of Mycobacterium tuberculosis. Microbiology 156, 2691-2701 (2010). and genetic program responsible for hepatic coenzyme A homeostasis. Chem. Biol. 14, 291-302 (2007).

22. Leonardi, R., Rehg, J. E., Rock, C. O. \& Jackowski, S. Pantothenate kinase 1 is required to support the metabolic transition from the fed to the fasted state. PLoS One 5, e11107 (2010).

24. Yocum, R. R., Patterson, T. A. \& Inc, O. B. Microorganisms and assays for the identification of antibiotics. U. S. Patent 6,830,898 (2004).

25. Brand, L. A. \& Strauss, E. Characterization of a new pantothenate kinase isoform from Helicobacter pylori. J. Biol. Chem. 280, 20185-20188 (2005).

26. Yao, J., Subramanian, C., Rock, C. O. \& Jackowski, S. Human pantothenate kinase 4 is a pseudo-pantothenate kinase. Protein Sci. 28, 1031-1047 (2019).

27. Tjhin, E. T. et al. Mutations in the pantothenate kinase of Plasmodium falciparum confer diverse sensitivity profiles to antiplasmodial pantothenate analogues. PLoS Pathog. 14, e1006918 (2018).

29. Sidik, S. M. et al. A genome-wide CRISPR screen in Toxoplasma identifies essential apicomplexan genes. Cell 166, 1423-1435.e12 (2016).

30. Zhang, M. et al. Uncovering the essential genes of the human malaria parasite Plasmodium falciparum by saturation mutagenesis. Science 360 , eaap7847 (2018). 
651 31. Aitken, A. 14-3-3 and its possible role in co-ordinating multiple signalling pathways. Trends Cell Biol. 6, 341-347 (1996).

32. Lalle, M. et al. Dematin, a component of the erythrocyte membrane skeleton, is internalized by the malaria parasite and associates with Plasmodium 14-3-3. J. Biol. Chem. 286, 1227-1236 (2011).

33. Brown, K. M., Long, S. \& Sibley, L. D. Plasma Membrane Association by N-Acylation Governs PKG Function in Toxoplasma gondii. MBio 8, F1000 (2017).

34. Rajendran, E. et al. Cationic amino acid transporters play key roles in the survival and transmission of apicomplexan parasites. Nat. Commun. 8, 14455 (2017).

38. Leonardi, R. et al. Modulation of pantothenate kinase 3 activity by small molecules that interact with the substrate/allosteric regulatory domain. Chem. Biol. 17, 892-902 (2010).

39. Subramanian, C. et al. Allosteric regulation of mammalian pantothenate kinase. J. Biol. Chem. 291, 22302-22314 (2016).

40. Crichton, P. G., Harding, M., Ruprecht, J. J., Lee, Y. \& Kunji, E. R. S. Lipid, detergent, and Coomassie Blue G-250 affect the migration of small membrane proteins in blue native gels: mitochondrial carriers migrate as monomers not dimers. J. Biol. Chem. 288, 22163-22173 (2013).

41. Al-Khedery, B., Barnwell, J. W. \& Galinski, M. R. Stage-specific expression of 14-3-3 in asexual blood-stage Plasmodium. Mol. Biochem. Parasitol. 102, 117-130 (1999).

42. Di Girolamo, F. et al. Plasmodium lipid rafts contain proteins implicated in vesicular trafficking and signalling as well as members of the PIR superfamily, potentially implicated in host immune system interactions. Proteomics 8, 2500-2513 (2008).

43. Dastidar, E. G. et al. Comprehensive histone phosphorylation analysis and identification of Pf14-3-3 protein as a histone H3 phosphorylation reader in malaria parasites. PLoS One 8, e53179 (2013). 
44. Rosenquist, M., Sehnke, P., Ferl, R. J., Sommarin, M. \& Larsson, C. Evolution of the 14-3-3 protein family: does the large number of isoforms in multicellular organisms reflect functional specificity? J. Mol. Evol. 51, 446-458 (2000).

45. van Hemert, M. J., Steensma, H. Y. \& van Heusden, G. P. 14-3-3 proteins: key regulators of cell division, signalling and apoptosis. Bioessays 23, 936-946 (2001).

46. Muslin, A. J. \& Xing, H. 14-3-3 proteins: regulation of subcellular localization by molecular interference. Cell. Signal. 12, 703-709 (2000).

47. Bridges, D. \& Moorhead, G. B. G. 14-3-3 proteins: a number of functions for a numbered protein. Sci. STKE 2005, re10-re10 (2005).

48. Saliba, K. J., Ferru, I. \& Kirk, K. Provitamin B5 (pantothenol) inhibits growth of the intraerythrocytic malaria parasite. Antimicrob. Agents Chemother. 49, 632-637 (2005).

49. Saliba, K. J. \& Kirk, K. CJ-15,801, a fungal natural product, inhibits the intraerythrocytic stage of Plasmodium falciparum in vitro via an effect on pantothenic acid utilisation. Mol. Biochem. Parasitol. 141, 129-131 (2005).

50. Spry, C., Chai, C. L. L., Kirk, K. \& Saliba, K. J. A class of pantothenic acid analogs inhibits Plasmodium falciparum pantothenate kinase and represses the proliferation of malaria parasites. Antimicrob. Agents Chemother. 49, 4649-4657 (2005).

51. Spry, C. et al. Pantothenamides are potent, on-target inhibitors of Plasmodium falciparum growth when serum pantetheinase is inactivated. PLoS One 8, e54974 (2013).

52. Fletcher, S. \& Avery, V. M. A novel approach for the discovery of chemically diverse anti-malarial compounds targeting the Plasmodium falciparum Coenzyme A synthesis pathway. Malar. J. 13, 343 (2014).

53. Saliba, K. J. \& Spry, C. Exploiting the coenzyme A biosynthesis pathway for the identification of new antimalarial agents: the case for pantothenamides. Biochem. Soc. Trans. 42, 1087-1093 (2014).

54. Macuamule, C. J. et al. A pantetheinase-resistant pantothenamide with potent, ontarget, and selective antiplasmodial activity. Antimicrob. Agents Chemother. 59, 3666-3668 (2015).

55. Pett, H. E. et al. Novel pantothenate derivatives for anti-malarial chemotherapy. Malar. J. 14, 169 (2015).

56. Howieson, V. M. et al. Triazole substitution of a labile amide bond stabilizes pantothenamides and improves their antiplasmodial potency. Antimicrob. Agents Chemother. 60, 7146-7152 (2016). 
57. Fletcher, S. et al. Biological characterization of chemically diverse compounds targeting the Plasmodium falciparum coenzyme A synthesis pathway. Parasit. Vectors 9, 589 (2016).

58. de Villiers, M. et al. Antiplasmodial mode of action of pantothenamides: pantothenate kinase serves as a metabolic activator not as a target. ACS Infect. Dis. 3, 527-541 (2017).

59. Weidner, T. et al. Antiplasmodial dihetarylthioethers target the coenzyme A synthesis pathway in Plasmodium falciparum erythrocytic stages. Malar. J. 16, 192 (2017).

60. Spry, C. et al. Structure-activity analysis of CJ-15,801 analogues that interact with Plasmodium falciparum pantothenate kinase and inhibit parasite proliferation. Eur. J. Med. Chem. 143, 1139-1147 (2018).

61. Guan, J. et al. Structure-Activity Relationships of Antiplasmodial Pantothenamide Analogues Reveal a New Way by Which Triazoles Mimic Amide Bonds. ChemMedChem 13, 2677-2683 (2018).

62. Warrenfeltz, S. et al. EuPathDB: The Eukaryotic Pathogen Genomics Database Resource. Methods Mol. Biol. 1757, 69-113 (2018).

63. Allen, R. J. \& Kirk, K. Plasmodium falciparum culture: The benefits of shaking. Mol. Biochem. Parasitol. 169, 63-65 (2010).

64. Jacot, D., Meissner, M., Sheiner, L., Soldati-Favre, D. \& Striepen, B. in Toxoplasma Gondii 577-611 (Elsevier, 2014). doi:10.1016/B978-0-12-396481-6.00017-9

65. Tjhin, E. T., Staines, H. M., van Schalkwyk, D. A., Krishna, S. \& Saliba, K. J. Studies with the Plasmodium falciparum hexokinase reveal that PfHT limits the rate of glucose entry into glycolysis. FEBS Lett. 587, 3182-3187 (2013).

66. Shen, B., Brown, K. M., Lee, T. D. \& Sibley, L. D. Efficient gene disruption in diverse strains of Toxoplasma gondii using CRISPR/CAS9. MBio 5, e01114-14 (2014).

67. van Dooren, G. G., Tomova, C., Agrawal, S., Humbel, B. M. \& Striepen, B. Toxoplasma gondii Tic20 is essential for apicoplast protein import. Proc. Natl. Acad. Sci. U.S.A. 105, 13574-13579 (2008).

68. Saliba, K. J., Horner, H. A. \& Kirk, K. Transport and metabolism of the essential vitamin pantothenic acid in human erythrocytes infected with the malaria parasite Plasmodium falciparum. J. Biol. Chem. 273, 10190-10195 (1998).

69. Somogyi, M. Determination of blood sugar. J. Biol. Chem. 160, 69-73 (1945).

70. Spry, C., Saliba, K. J. \& Strauss, E. A miniaturized assay for measuring small molecule phosphorylation in the presence of complex matrices. Anal. Biochem. 451, 76-78 (2014). 
753 71. Pei, J. \& Grishin, N. V. PROMALS3D: multiple protein sequence alignment enhanced with evolutionary and three-dimensional structural information. Methods Mol. Biol. 1079, 263-271 (2014). 


\section{Supplementary Information}

\section{A novel heteromeric pantothenate kinase complex in apicomplexan parasites Erick T. Tjhin, Vanessa M. Howieson, Christina Spry, Giel G. van Dooren and Kevin J. Saliba}

\section{METHODS}

\section{Plasmid preparation}

The Pfpank2-pGlux-1 construct has the Pfpank2-coding sequence inserted within multiple cloning site (MCS) III of pGlux-1. The plasmid backbone contains the human dihydrofolate reductase (hdhfr) gene, which confers resistance to WR99210, as a positive selectable marker. Pfpank2 is placed under the regulation of the Plasmodium falciparum chloroquine resistance transporter (Pfcrt) promoter, and upstream of the GFP-coding sequence.

The Pfpank2 sequence used to generate the Pfpank2-pGlux-1 construct was initially amplified from parasite cDNA. Total RNA was purified from saponin-isolated $P$. falciparum parasites (typically $2 \times 10^{7}$ cells) using the RNeasy Mini Kit (QIAGEN) according to the manufacturer's protocol for purifying total RNA from animal cells. The optional $15 \mathrm{~min}$ DNase I incubation was included to eliminate residual genomic DNA. Complementary DNA (cDNA) was synthesised from this total RNA sample using SuperScript II Reverse Transcriptase (ThermoFisher) with Oligo(dT) ${ }_{12-18}$ primer and included the optional incubation with RNaseOUT Recombinant Ribonuclease Inhibitor (ThermoFisher), all according to the manufacturer's protocol. The Pfpank2-specific sequence was then amplified from cDNA using Platinum Pfx DNA polymerase (ThermoFisher) with the oligonucleotide primers listed in Table S1. The Pfpank2-coding sequence was then inserted into pGlux-1 using the InFusion cloning (Clontech) method. Before cloning, the pGlux-1 plasmid was linearised by sequential digestions with Xhol (ThermoFisher) and subsequently Kpnl (New England Biolabs), according to each manufacturer's recommendation. The In-Fusion reaction was set up with the In-Fusion Dry-Down PCR Cloning Kit, essentially as described in the manufacturer's protocol.

The TgPanK1-mAIDHA and TgPank2-mAIDHA expressing lines were generated using a CRISPR/Cas9 based genome editing approach as previously described in Shen et al. 1. Guide RNA (gRNA) sequences that would enable Cas9 to complex with the RNA to cut close to the 3' end of either the Tgpank1 or Tgpank2 gene were incorporated into pSAG1::Cas9-U6::sgUPRT (Addgene plasmid $\# 54467{ }^{1}$ ) by utilising the Q5 site-directed 
mutagenesis kit (New England Biolabs). This included performing an initial PCR incorporating the gRNA into the vector and subsequently circularising the vector. This vector encodes both the gRNA and the Cas9-GFP. A gBlock (IDT) containing the mAIDHA construct ${ }^{2}$ was also amplified using gene-specific primers with homologous ends to the locus targeted by the gRNA to enable homologous recombination. The gRNA-expressing pSAG1::CAS9-U6::sgUPRT construct for each Tgpank gene was transfected with the corresponding mAIDHA gBlock fragment into $\mathrm{RH}$ strain TATi $\triangle$ Ku80:TIR1 tachyzoite-stage parasites ${ }^{2}$ also expressing a tandem dimeric (td) Tomato red fluorescent protein. The GFP expression from the Cas9-GFP enabled the use of flow cytometry to select GFP positive cells to establish a clonal population two days after transfection. Transformants were identified by PCR screening (all primers and gBlocks are listed in Table S1).

The TgPanK1-GFP/TgPanK2-mAIDHA expressing line was generated utilising the CRISPR/Cas9 approach as mentioned above. A gBlock (IDT) containing the TEV-GFP construct was amplified using gene-specific primers with homologous ends to the Tgpank1 locus targeted by the gRNA to enable homologous recombination. This was transfected with the gRNA-expressing pSAG1::CAS9-U6::sgUPRT construct for Tgpank1 into the TgPanK2mAIDHA line that had been previously created. GFP positive cells were selected for as noted above and a clonal line was confirmed by PCR screening (all primers and gBlock are listed in Table S1). The TgPanK1-HA/TgPanK2-GFP expressing line was generated in RH strain $\mathrm{TATi} \Delta \mathrm{Ku} 80$ parasites using the same CRISPR/Cas9 approach with the TEV-HA gBlock and TEV-GFP gBlock (IDT).

The complementation lines TgPanK1-mAIDHA ${ }^{+S a P a n K-T y 1}$ and TgPanK2-mAIDHA ${ }^{+S a P a n K-T y 1}$ were created by expressing Ty1-tagged Staphylococcus aureus type II pantothenate kinase (Sapank) in the TgPanK1-mAIDHA and TgPanK2-mAIDHA T. gondii strains. Briefly, the Sapank open reading frame was PCR amplified using a gBlock encoding Sapank that had been codon-optimised for T. gondii expression. The resultant PCR product was digested with Bg/ll and Avrll and ligated into the equivalent sites of the vector pUBTTY. The pUBTTy was modified from the vector PBTTy, described previously ${ }^{3}$, which contains a phleomycin resistance marker, and an expression cassette containing the $T$. gondii $\alpha$-tubulin promoter and a Ty1 epitope tag. The UPRT flank was digested from the vector pUgCTH3 ${ }^{4}$ with Apal and HindIII and ligated into the equivalent sites of pBTTy to generate pUBTTy. The SapankTy1 containing pUBTTy vector was linearised and transfected into the lines expressing TgPanK1-mAIDHA and TgPanK2-mAIDHA. Transformants were subsequently selected 
using phleomycin $(50 \mu \mathrm{g} / \mathrm{mL})$ in DMEM supplemented with $10 \mathrm{mM}$ Hepes and $10 \mu \mathrm{g} / \mathrm{mL}$ gentamicin, $\mathrm{pH} 7.6$, for $4 \mathrm{~h}$ as described previously ${ }^{5}$. Phelomycin-resistant parasites were cloned using fluorescence activated cell sorting, and subsequently cultured in complete RPMI-1640.

\section{Preparation of cells for microscopy}

Coverslip-bound $P$. falciparum infected red blood cells were first prepared by washing (500 $\times g, 5$ min) parasite-infected erythrocytes (5 - 10\% parasitaemia) once and resuspending them at $\sim 2 \%$ haematocrit in $137 \mathrm{mM} \mathrm{NaCl}, 2.7 \mathrm{mM} \mathrm{KCl}, 10 \mathrm{mM}$ phosphate buffer, $\mathrm{pH} 7.4$ (phosphate buffered saline; PBS). Next, $1-2 \mathrm{~mL}$ of the suspension was added to a polyethylenimine (PEI)-coated coverslip placed within a well of a 6-well plate. Plates were incubated (with shaking) for $15 \mathrm{~min}$ at room temperature and unbound cells were subsequently washed off the coverslips with PBS $(2 \mathrm{~mL}$ per well, with a 2 min shaking incubation followed by aspiration). Cells were then fixed with $1 \mathrm{~mL}$ of PBS containing $4 \%$ (w/v) paraformaldehyde (Electron Microscopy Services) and 0.0075\% (w/v) glutaraldehyde (30 min at room temperature). The fixative was then aspirated, and the coverslips washed in PBS three times as described above, before they were rinsed in water and dried. A drop of SLOWFADE (Invitrogen) containing the nuclear stain 4',6-diamidino-2-phenylindole (DAPI) was added to the centre of the coverslips. Finally, each coverslip was inverted onto a microscope slide, sealed with nail polish and used for confocal imaging.

For T. gondii immunofluorescence assays, parasites were inoculated onto HFF-coated coverslips and allowed to proliferate overnight. Parasites were fixed in $3 \%(\mathrm{w} / \mathrm{v})$ paraformaldehyde in PBS, permeabilised in 0.25\% (v/v) Triton X-100 in PBS and blocked in $2 \%(\mathrm{w} / \mathrm{v})$ bovine serum albumin in PBS. Parasites were incubated in mouse anti-Ty1 primary antibodies (1:200 dilution; ${ }^{6}$ ) and goat anti-mouse AlexaFluor 488-conjugated secondary antibodies (1:250 dilution; ThermoFisher, catalogue number A11029).

\section{Denaturing polyacrylamide gel electrophoresis}

Saponin-isolated $P$. falciparum parasites (typically $\sim 10^{8}$ cells) were centrifuged $(15,850 \times g$, $30 \mathrm{~s}$ ) and the supernatant was removed. The pellet was resuspended in $200 \mu \mathrm{L}$ of lysis buffer $(1 \times$ mini cOmplete protease inhibitor cocktail (Roche), $1 \times$ NuPAGE LDS sample buffer (ThermoFisher), $1 \times$ NuPAGE sample reducing agent (ThermoFisher), $50-60$ units of benzonase nuclease (Novagen) and 7.5 - $10 \mathrm{mM}$ of $\mathrm{MgCl}_{2}$ ), mixed well by vortexing and 
then incubated at $95^{\circ} \mathrm{C}$ for $10 \mathrm{~min}$. The sample was then centrifuged $(16,000 \times \mathrm{g}, 30 \mathrm{~min})$ to pellet the haemozoin before the supernatant was used for gel electrophoresis. Each sample $(10 \mu \mathrm{L})$ was subsequently loaded into separate wells of a NuPAGE $4-12 \%$ Bis-Tris protein gel $(1.0 \mathrm{~mm}, 12$ wells; ThermoFisher) alongside $5 \mu \mathrm{L}$ of SeeBlue Plus2 pre-stained protein standards (ThermoFisher).

$T$. gondii tachyzoites, either freshly egressed from host HFF cells or mechanically egressed through a 26-gauge needle, were filtered through a $3 \mu \mathrm{m}$ polycarbonate filter. Tachyzoites (typically $1.5 \times 10^{7}$ cells), were subsequently centrifuged $(12,000 \times \mathrm{g}, 1 \mathrm{~min})$. The supernatant was aspirated and the pellet was resuspended in $30 \mu \mathrm{L}$ of $1 \times$ NuPAGE LDS sample buffer (ThermoFisher). The sample was mixed well by vortexing and incubated at $95{ }^{\circ} \mathrm{C}$ for $10 \mathrm{~min}$. Samples were then frozen at $-20{ }^{\circ} \mathrm{C}$ or used immediately for gel electrophoresis. Each sample $(10-20 \mu \mathrm{L})$ was subsequently loaded into separate wells of a NuPAGE $4-12 \%$ Bis-Tris protein gel $(1.0 \mathrm{~mm}, 12$ wells; ThermoFisher) alongside $5 \mu \mathrm{L}$ of Novex Sharp Pre-Stained Protein Standard (Invitrogen). Where relevant, parasites were incubated in $100 \mu \mathrm{M}$ idole-3-acetic acid (IAA) or in a $0.1 \%(\mathrm{v} / \mathrm{v})$ ethanol vehicle control for specified times prior to sample preparation.

Electrophoresis of $P$. falciparum and $T$. gondii samples was performed in $1 \times$ NUPAGE 2( $N$-morpholino)ethanesulfonic acid (MES) sodium dodecyl sulfate (SDS) running buffer (ThermoFisher) at $200 \mathrm{~V}$ for $30-35 \mathrm{~min}$. Separated proteins were transferred ( $35 \mathrm{~V}$ for 1.5 $\mathrm{h}$ or $30 \mathrm{~V}$ for $1 \mathrm{~h}$ depending on the transfer system) to a nitrocellulose membrane (ThermoFisher) in $1 \times$ NuPAGE transfer buffer containing $10 \%(\mathrm{v} / \mathrm{v})$ methanol. The membrane was then blocked in a solution containing $4 \%(\mathrm{w} / \mathrm{v})$ skim milk powder in Tris buffered saline (TBS) or PBS with shaking, either overnight at $4{ }^{\circ} \mathrm{C}$ or for $1-2 \mathrm{~h}$ at room temperature.

The primary antibodies used in this study included mouse anti-GFP monoclonal antibody $(0.4 \mathrm{\mu g} / \mathrm{mL}$ final concentration; Roche, Sigma catalogue 11814460001), rat anti-HA monoclonal antibody $(1.6 \mu \mathrm{g} / \mathrm{mL}$ final concentration; Sigma, clone $3 \mathrm{~F} 10$, catalogue A11867431001), mouse anti-Ty1 monoclonal antibody (1:1000 dilution; $\left.{ }^{6}\right)$, and a panspecific anti-14-3-3 rabbit polyclonal antibody $(0.25 \mu \mathrm{g} / \mathrm{mL}$ final concentration; Abcam). The secondary antibodies used for the $P$. falciparum blots were a goat anti-mouse horseradish peroxidase (HRP)-conjugated antibody and a goat anti-rabbit HRP-conjugated antibody (both $0.08 \mu \mathrm{g} / \mathrm{mL}$ final concentration; Santa Cruz Biotechnology). The secondary antibodies 
used for T. gondii experiments were goat anti-mouse HRP-conjugated antibody $(0.4-0.8$ $\mu \mathrm{g} / \mathrm{mL}$ final concentration; Abcam), goat anti-rabbit HRP-conjugated antibody $(0.1 \mu \mathrm{g} / \mathrm{mL}$ final concentration; Abcam), and goat anti-rat HRP-conjugated antibody $(0.1 \mu \mathrm{g} / \mathrm{mL}$ final concentration; Abcam).

All antibodies were diluted in $4 \%(w / v)$ skim milk in TBS or PBS. After each antibody incubation, membranes were washed at least three times (5 - $10 \mathrm{~min}$ each) in fresh 0.05 or $0.1 \%(v / v)$ Tween 20 in TBS or PBS.

\section{Native polyacrylamide gel electrophoresis}

In order to detect the presence and abundance of protein(s) of interest in their native conformation, parasite samples were subjected to blue native gel electrophoresis. Briefly, saponin-isolated trophozoite-stage $P$. falciparum parasites $\left(4-8 \times 10^{8}\right.$ cells) were centrifuged $(15,850 \times g, 30 \mathrm{~s})$ and the supernatant removed from the pellet. The parasites were then resuspended by vortexing in $200 \mu \mathrm{L}$ of lysis buffer containing $1 \times$ mini cOmplete protease inhibitor cocktail (Roche), $1 \times$ NativePAGE sample buffer (ThermoFisher), 0.5\% (w/v) digitonin, $2 \mathrm{mM}$ EDTA, 50 - 60 units of benzonase nuclease (Novagen) and $7.5-10$ $\mathrm{mM}$ of $\mathrm{MgCl}_{2}$, and incubated with tumbling end-over-end at $4{ }^{\circ} \mathrm{C}$. The lysis preparation was then centrifuged at $16,000 \times g$ for $30 \mathrm{~min}$ at $4{ }^{\circ} \mathrm{C}$ and the supernatant immediately used for gel electrophoresis. Prior to electrophoresis, NativePAGE 5\% G-250 sample additive (ThermoFisher) was added to each sample supernatant to a final concentration of $0.125 \%$ $(w / v)$ and mixed by vortexing. Samples (typically $10 \mu \mathrm{L}$ ) were then loaded into the wells of a NativePAGE 4 - 16\% Bis-Tris protein gel (1.0 mm, 10 wells; ThermoFisher). NativeMark unstained protein standards ( $5 \mu \mathrm{L}$, ThermoFisher) were loaded into the gel alongside the samples to allow for protein mass determination. Electrophoresis was carried out at $4{ }^{\circ} \mathrm{C}$ according to the manufacturer's protocol for detergent-containing samples to be used for western blotting. At the end of the run, the proteins within the gel were transferred as described for denaturing western blot above to a methanol-primed $0.45 \mu \mathrm{m}$ PVDF membrane (GE healthcare). At the end of the transfer, proteins were fixed to the membrane by a 15 min incubation in $10 \%(\mathrm{v} / \mathrm{v})$ acetic acid and briefly rinsed in water. In order to visualise the ladder, the membrane was very briefly ( $\sim \mathrm{s})$ de-stained in absolute methanol and rinsed in water before it was blocked overnight in $4 \%(\mathrm{w} / \mathrm{v})$ skim milk powder in PBS at $4{ }^{\circ} \mathrm{C}$ with shaking. 
T. gondii tachyzoites freshly or mechanically egressed from their host HFF cells were filtered through a $3 \mu \mathrm{m}$ polycarbonate filter. Tachyzoites (typically $1.5 \times 10^{7}$ cells) were subsequently centrifuged $(12,000 \times g, 1 \mathrm{~min})$. The supernatant was removed and the pellet was resuspended by vortexing in $30 \mu \mathrm{L}$ of lysis buffer containing $1 \times$ mini cOmplete protease inhibitor cocktail (Roche), $1 \times$ NativePAGE sample buffer (ThermoFisher), $10 \%(\mathrm{v} / \mathrm{v}$ ) Triton $\mathrm{X}-100,2 \mathrm{mM}$ EDTA, and incubated with intermittent perturbation on ice for $30 \mathrm{~min}$. The lysate was then centrifuged at $20,000 \times g$ for 30 min at $4{ }^{\circ} \mathrm{C}$ and the supernatant was either stored at $-20{ }^{\circ} \mathrm{C}$ or used immediately for gel electrophoresis. Sample preparation, electrophoresis and transfer was carried out as described for $P$. falciparum. Where anti-HA blotting was required on a PVDF membrane, after electrophoresis of the samples the membrane was placed in TBS containing $0.05 \%$ (v/v) Tween 20 overnight, then blocked in $4 \%(\mathrm{w} / \mathrm{v})$ skim milk powder in TBS at room temperature for a minimum of $1 \mathrm{~h}$ the next day before probing with the anti HA antibody.

\section{Immunoprecipitation}

Briefly, saponin-isolated $P$. falciparum trophozoites were resuspended in $500 \mu \mathrm{L}$ of lysis buffer containing $1 \times$ mini cOmplete protease inhibitor cocktail (Roche) or $1 \times$ Halt protease inhibitor cocktail (EDTA-free; ThermoFisher), GFP-Trap wash buffer (10 mM Tris/Cl, pH 7.5, $150 \mathrm{mM} \mathrm{NaCl}$ and $0.5 \mathrm{mM}$ EDTA), 0.5\% (w/v) digitonin, 50 - 60 units of benzonase nuclease (Novagen) and $3-4 \mathrm{mM}$ of $\mathrm{MgCl}_{2}$. The pellet was resuspended well by vortexing and the suspension was incubated (30 - $60 \mathrm{~min}$ ) with tumbling end-over-end at $4{ }^{\circ} \mathrm{C}$. Subsequently, the suspension was centrifuged $\left(16,000 \times g, 30 \mathrm{~min}, 4{ }^{\circ} \mathrm{C}\right)$ and the supernatant used for GFP-Trap binding. Prior to immunoprecipitation, $25 \mu \mathrm{L}$ (for each lysate) of GFP-Trapagarose bead slurry was primed by three washes $\left(2,500 \times \mathrm{g}, 2 \mathrm{~min}, 4^{\circ} \mathrm{C}\right)$ in $500 \mu \mathrm{L}$ of GFP. Trap wash buffer. The supernatant was removed from the beads at the end of the third wash and $450-500 \mu \mathrm{L}$ of the lysate generated in the parasite lysis step was applied to the beads. In some experiments, $50 \mu \mathrm{L}$ of the total lysate was collected for western blotting. This suspension was then incubated for $1 \mathrm{~h}$ at $4{ }^{\circ} \mathrm{C}$ with tumbling end-over-end. At the end of the incubation, the bead suspension was centrifuged $\left(2,500 \times g, 2 \mathrm{~min}, 4{ }^{\circ} \mathrm{C}\right)$ and the supernatant was discarded. In some experiments, $50 \mu \mathrm{L}$ of this supernatant was collected to be used in western blots as the unbound fraction. The proteins bound to the beads were washed $3 \times\left(2,500 \times g, 2 \mathrm{~min}, 4^{\circ} \mathrm{C}\right)$ in GFP-Trap wash buffer with or without $1 \times \operatorname{mini}$ cOmplete protease inhibitor cocktail or $1 \times$ Halt protease inhibitor cocktail (EDTA-free). After removing the supernatant at the end of the third wash, the beads were resuspended in GFP. 
Trap wash buffer (typically $200-300 \mu \mathrm{L}$ ) and aliquots of these were used for downstream experiments.

T. gondii GFP-tagged proteins were purified using the GFP-Trap approach mentioned above, and the HA-tagged proteins were purified using anti-HA Affinity Matrix (Roche) following the manufacturer's instructions with some modifications. Egressed T. gondii tachyzoites were filtered through a $3 \mu \mathrm{m}$ polycarbonate filter. Parasites $\left(\sim 10^{7}-10^{8}\right.$ for each line) were centrifuged $\left(1,500 \times g, 10 \mathrm{~min}, 4^{\circ} \mathrm{C}\right)$. The supernatant was aspirated, and the cells were resuspended in $1 \mathrm{~mL}$ of PBS and centrifuged $\left(12,000 \times \mathrm{g}, 1 \mathrm{~min}, 4^{\circ} \mathrm{C}\right)$. After aspiration, lysis buffer $(1 \mathrm{~mL})$ containing $1 \times$ mini cOmplete protease inhibitor cocktail (Roche), wash buffer (10 mM Tris/Cl, pH 7.5, $150 \mathrm{mM} \mathrm{NaCl}$ and $0.5 \mathrm{mM}$ EDTA), and 1\% (v/v) Triton X-100 was added to the remaining cells. The pellet was resuspended and the suspension was incubated $(1 \mathrm{~h})$ with tumbling end-over-end at $4{ }^{\circ} \mathrm{C}$. Subsequently, the suspension was centrifuged $\left(21,000 \times g, 30 \mathrm{~min}, 4^{\circ} \mathrm{C}\right)$. Prior to immunoprecipitation, $25 \mu \mathrm{L}$ of GFP-Trapagarose bead slurry, or $25 \mu \mathrm{L}$ of anti-HA Affinity Matrix were washed three times in $500 \mu \mathrm{L}$ of wash buffer (with 1\% (v/v) Triton X-100 in the anti-HA wash buffer), with centrifugation at $2,500 \times g, 2 \mathrm{~min}, 4^{\circ} \mathrm{C}$ between washes. The supernatant was removed from the beads after the third wash and $450 \mu \mathrm{L}$ of the parasite lysate was applied to each of the beads. A $50 \mu \mathrm{L}$ aliquot of the total lysate was collected for western blotting as the 'total' fraction. The lysate/bead suspension was incubated for at least $1 \mathrm{~h}$ at $4{ }^{\circ} \mathrm{C}$ with tumbling end-over-end. At the end of the incubation, the bead suspension was centrifuged $\left(2,500 \times g, 2 \mathrm{~min}, 4{ }^{\circ} \mathrm{C}\right)$, $50 \mu \mathrm{L}$ of this supernatant was collected for use in western blots as the 'unbound' fraction. The proteins bound to the beads were washed $3 \times$ in wash buffer with centrifugation at $2,500 \times g, 2 \mathrm{~min}, 4^{\circ} \mathrm{C}$ between washes. After removing the supernatant at the end of the third wash, the beads were resuspended in wash buffer (typically $100-300 \mu \mathrm{L}$ ) and aliquots of these were used for downstream experiments. Alternatively, $100 \mu \mathrm{L}$ of $1 \times$ NuPAGE LDS sample buffer (ThermoFisher) was added to the samples to elute proteins from the beads and generate the 'bound' fractions.

\section{Mass spectrometry of immunoprecipitated samples}

The immunoprecipitated proteins were processed and identified through mass spectrometry (MS) analysis at the Australian Proteomics Analysis Facility. First, the loading buffer in the samples were separated from the proteins through a short one-dimension gel electrophoresis. The samples were denatured at $95{ }^{\circ} \mathrm{C}$ for $10 \mathrm{~min}$ and $2 \times 15 \mu \mathrm{L}$ of each 
sample was loaded into the lanes of a $12 \%$ iGel protein gel (1.0 mm, 12-well; NuSep). The gel was run at $15 \mathrm{~mA}$ for $25 \mathrm{~min}$ and subsequently washed with a solution containing $10 \%$ (v/v) methanol and 7\% (v/v) acetic acid for $15 \mathrm{~min}$. The gel was then washed with a fixant for 90 min and stained overnight in Coomassie. The band corresponding to the proteins was subsequently excised and de-stained with ammonium bicarbonate/acetonitrile (ACN). The protein samples were then reduced with $25 \mathrm{mM}$ dithiothreitol (DTT) at $60{ }^{\circ} \mathrm{C}$ for $30 \mathrm{~min}$ and alkylated with $55 \mathrm{mM}$ iodoacetamide before an in-gel protein digestion was performed overnight using $200 \mathrm{ng}$ trypsin. The peptides generated were extracted from the gel with bath sonication and ACN/formic acid (FA), dried and then reconstituted in $30 \mu \mathrm{L}$ of loading buffer.

The peptide samples were then subjected to 1D nano liquid chromatography tandem mass spectrometry (Nano-LC-ESI MS/MS) analysis. Sample $(10 \mu \mathrm{L})$ was injected onto a peptide trap (Halo C18, $150 \mu \mathrm{m} \times 5 \mathrm{~cm}$ ) for pre-concentration and desalted with $0.1 \%(\mathrm{v} / \mathrm{v}) \mathrm{FA}, 2 \%$ $(\mathrm{v} / \mathrm{v}) \mathrm{ACN}$ at $4 \mu \mathrm{L} / \mathrm{min}$ for $10 \mathrm{~min}$. The peptide trap was then switched into line with the analytical column. Peptides were subsequently eluted from the column using a linear solvent gradient, with steps, from $\mathrm{H}_{2} \mathrm{O}: \mathrm{ACN}\left(98: 2 ;+0.1 \%\right.$, v/v, FA) to $\mathrm{H}_{2} \mathrm{O}: \mathrm{CH}_{3} \mathrm{CN}(2: 98 ;+0.1 \%$, $\mathrm{v} / \mathrm{v}, \mathrm{FA})$ with constant flow (600 $\mathrm{nL} / \mathrm{min})$ over an $80 \mathrm{~min}$ period. The liquid chromatography eluent was subjected to positive ion nanoflow electrospray MS analysis in an informationdependent acquisition mode (IDA). In the IDA mode, a time-of-flight MS survey scan was acquired ( $\mathrm{m} / \mathrm{z} 350-1500,0.25 \mathrm{~s}$ ), with twenty largest multiply charged ions (counts $>150$ ) in the survey scan sequentially subjected to MS/MS analysis. MS/MS spectra were accumulated for $100 \mathrm{~ms}(\mathrm{~m} / \mathrm{z} 100-1500)$ with rolling collision energy.

The peptides from the MS analysis were identified by comparing their amino acid sequences against an annotated protein database for $P$. falciparum 3D7 strain (version 28; PlasmoDB) using the ProteinPilot Software (version 4.2; SCIEX) at a detection threshold of $>1.30(95.0 \%$ confidence).

\section{Fluorescent parasite proliferation assay}

Fluorescent parasite proliferation assays were performed as previously described ${ }^{4,7}$. Black 96-well plates containing confluent HFF host cells were washed with PBS $(\times 2)$. Complete RPMI-1640 (100 $\mu \mathrm{L})$ with or without $200 \mu \mathrm{M}$ of IAA supplementation $(2 \times$ final concentration), or with $10 \mu \mathrm{M}$ pyrimethamine $(2 \times$ final concentration) for the 'no growth' control, were added 
to the relevant wells. Fluorescent parasites (parental line, TgPanK1-mAIDHA, TgPanK2mAIDHA, TgPanK1-mAIDHA ${ }^{+S a P a n K-T y 1}$ or TgPanK2-mAIDHA ${ }^{+S a P a n K-T y 1}$ ) were plated in each well $\left(100 \mu \mathrm{L}, 2000\right.$ parasites) in triplicate. Plates were incubated at $37{ }^{\circ} \mathrm{C}$ in a $5 \% \mathrm{CO}_{2}$ humidified incubator. Fluorescent measurements (Excitation filter, 540nm; Emission filter, $590 \mathrm{~nm}$ ) were taken up to two times a day over 7 days with the FLUOstar OPTIMA Microplate Reader (BMG LABTECH), and the proliferation of the fluorescent parasites was measured over this time. Values for the no growth control were considered as background values and were subtracted from the experimental values during data processing.

\section{Alignment of PanK}

The following PanK type II homologues annotated by accession number were aligned: Staphylococcus aureus (Q2FWC7), Saccharomyces cerevisiae (Q04430), Aspergillus nidulans (O93921), Homo sapiens PanK1 (Q8TE04) PanK2 (Q9BZ23) PanK3 (Q9H999) PanK4 (Q9NVE7), Arabidopsis thaliana PanK1 (O80765) PanK2 (Q8L5Y9), Plasmodium falciparum PanK1 (Q8ILP4) PanK2 (Q8IL92) and Toxoplasma gondii PanK1 (A0A125YTW9) PanK2 (V5B595). We utilised PROMALS3D 8 (available at: http://prodata.swmed.edu/promals3d/promals3d.php) for the alignment. 
bioRxiv preprint doi: https://doi.org/10.1101/2021.03.16.435557; this version posted March 16,2021 . The copyright holder for this preprint (which was not certified by peer review) is the author/funder, who has granted bioRxiv a license to display the preprint in perpetuity. It is made available under aCC-BY-NC-ND 4.0 International license.

Table S1. List of oligonucleotides used in this study.

\section{Oligonucleotide Name Sequence (5'-3')}

Pfpank2-pGlux-1-5'primer TTACATATAACTCGAGATGGGTAATACATTAGGTATTG

Pfpank2-pGlux-1-3'primer TCTTCTCCTTTACTGGTACCATTGTCTATGTGAATTCGTTCC

Pfpank2-internal-5'primer1 TCTGATGAATATAATAACTGTGATGACG

Pfpank2-internal-5'primer2 ATGAAAGAGCTAGCTGTACTAGCC

Pfpank2-internal-3'primer1 TCTTCGTCATTTTGGCTAGTACAGC

Pfpank2-internal-3'primer2 TCACTAATTTCTGTACTCATACAACTGC

Pfpank2-internal-3'primer3

ACCATTCTCATTTAGATATTGC

Pfpank2-internal-3'primer4 TCTGGATAAATCACACATTTTGG

Tgpank1-pSAG1::CAS9-U6::sgUPRT-3'primer AACTTCCCCTTGTCTAGCAGGTTTTAGAGCTAGAAATAGCAAG

Tgpank1 pSAG1::CAS9-U6::sgUPRT screening primer CTGCTAGACAAGGGGAAGTT

Tgpank1-mAID-5'primer TTGGAGCTCTGGTTGCTGCCGACGAATGCCCCGTGGAAAAATCICGC TGCGGTGGAGGTAGCGGTGGTGGAAG

Tgpank1-mAIDHA-3'primer

AGGGTCTCGAATGGACTCGAATGATGGCAAACGACGCAACTTCCCC

TTGTGCTTCTGTGGGCGGTTATCAGG

Tgpank1 3' internal-screen fwd

TTTCATCTGTATGGGACAGTGG

Tgpank1 3' internal-screen rev

CTCCAAGTCTACGTCACACCAC

Tgpank2-pSAG1::CAS9-U6::sgUPRT-3'primer

CTTCTCTGGCAGGAAGAACGGTTTTAGAGCTAGAAATAGCAAG

Tgpank2 pSAG1::CAS9-U6::sgUPRT screening primer CGTTCTTCCTGCCAGAGAAG

Tgpank2-mAID-5'primer

CTCCGCCATTTTTACCTCCTCTCGCCTCCTGCTTLCACGTTCTTCCTG CCAGAGAAGAGAGCGAGGGTGGAGGTAGCGGTGGTGGAAG

Tgpank2-mAIDHA-3'primer

TCGTTCTTGACATCTCCGCGCTGCACGCCTTTCCACCTGTTCCTCGT CGTGCTTCTGTGGGCGGTTATCAGG

Tgpank2-3' internal-screen fwd

AAGAGCCTCAGGAGGAGACG

Tgpank2-3' internal-screen rev

CGATCTCAACCTCCCACTTCT

\section{Sapank-pUDTTy-5'primer}

GATCAGATCTAAAATGAAGGTTGGAATTGATGCCG

Sapank-pUDTTy-3'primer

GCATCCTAGGCTTTTCAAGATAGAGTGCTCCGATC

\section{Function}

PCR amplification of Pfpank2 flanked by a 16-base sequence homologous with the linearisation site of pGlux-1 (in bold) that includes the Xhol restriction site (underlined). The transcription start codon is highlighted in blue.

PCR amplification of Pfpank2 flanked by a 20 -base sequence homologous with the linearisation site of pGlux-1 (in bold) that includes the Kpnl restriction site (underlined).

Sanger sequencing of Pfpank2-pGlux-1 clones.

Sanger sequencing of Pfpank2-pGlux-1 clones.

Sanger sequencing of Pfpank2-pGlux-1 clones.

Sanger sequencing of Pfpank2-pGlux-1 clones.

Sanger sequencing of Pfpank2-pGlux-1 clones.

Sanger sequencing of Pfpank2-pGlux-1 clones.

PCR amplification of gRNA targeting TgpanK1 near the stop codon flanked by a homologous region with pSAG1::CAS9-U6::sgUPRT (23 bp) underlined.

Sanger sequencing of Tgpank1-gRNA-pSAG1::CAS9-U6::sgUPRT clone.

PCR amplification of mAID coding sequence (underlined) flanked by Tgpank1 homologous region (50 bp) at the CRISPR/Cas9 cut site. Incorporation of 't' interrupts the original PAM site sequence.

PCR amplification of mAID $3 \times$ HA coding sequence (underlined) flanked by Tgpank1 homologous region (50 bp) at the CRISPR/Cas9 cut site.

Screening and Sanger sequencing of Tgpank1-mAIDHA clones

Screening and Sanger sequencing of Tgpank1-mAIDHA clones

PCR amplification of gRNA targeting Tgpank2 near the stop codon, flanked by a homologous region with pSAG1::CAS9-U6::sgUPRT (23 bp) underlined.

Sanger sequencing of Tgpank2-gRNA- pSAG1::CAS9-U6::sgUPRT clone.

PCR amplification of mAID coding sequence (underlined) flanked by PanK1 homologous region (65 bp) at the CRISPR/Cas9 cut site. Incorporation of 't' interrupts the original PAM site sequence.

PCR amplification of mAID coding sequence (underlined) flanked by Tgpank2 homologous region (50 bp) at the CRISPR/Cas9 cut site.

Screening and Sanger sequencing of Tgpank2-mAIDHA clones

Screening and Sanger sequencing of Tgpank2-mAIDHA clones

PCR amplification of codon optimised Sapank flanked by the Bglll restriction site (underlined), the transcription start codon is highlighted in blue.

PCR amplification of codon optimised Sapank flanked by the Avrll restriction site (underlined) 
bioRxiv preprint doi: https://doi.org/10.1101/2021.03.16.435557; this version posted March 16, 2021. The copyright holder for this preprint (which was not certified by peer review) is the author/funder, who has granted bioRxiv a license to display the preprint in perpetuity. It is made available under aCC-BY-NC-ND 4.0 International license.

\section{Table S2. List of gBLOCK sequences used in this study.}

\section{gBlock sequences}

Sapank (ORF optimised for expression in T. gondii at https://sg.idtdna.com/codonopt)

ATGAAGGTTGGAATTGATGCCGGAGGTACACTCATCAAAATCGTCCAGGAACAAGATAACCAGAGGACATTCAAGACAGAACTTACAAAAAACATCGACCAAGTCGTTGAGTGGCTCAATCAGCAGCAGA TCGAGAAGCTTTGCCTGACAGGTGGAAATGCTGGT GATCTGGCTGACTATATCTTCGCAAATGTTGGTACTGGTACTTCTCTCCATTATTTCGATGGGCAAAGCCAAAGACGAGTTGGAGGCATTGGTACTGGTGGCGGGATGATCCAGGGCCTGGGTTACCTCC TGAGTCAGATTACCGATTACAAACAGCTTACTGATATGGCTCAGCATGGAGACCGTAACACAATCGATCTGAAAGTTCGGCATATTTATAAAGACACCGAACCGCCAATCCCTGGTGATCTTACGGCCGCT AATTTCGGCCATGTCCTGCATCATCTTGATGCCGACTTCACGCCTTCGAATAAGCTCGCCGCCGTTATCGGGGTTGTGGGGGAGGTTGTTACAACAATGGCTATTACAGTTGCGAGGGAGTTCAAAACGG AGAACATTGTGTATATTGGCTCCAGCTTCCACAATAATGCACTCCTTCGTAAAGTGGTTGAGGACTATACAGTGCTGCGTGGTTGTAAGCCCTACTATGTGGAGAATGGCGCATTTTCTGGTGCGATCGGA GCACTCTATCTTGAAAAG

mAID

GGTGGAGGTAGCGGTGGTGGAAGTGAGAAGAGCGCGTGTCCTAAAGATCCCGCTAAGCCGCCTGCCAAGGCCCAGGTGGTTGGCTGGCCCCCGGTTAGGAGTTACCGCAAGAACGTGATGGTCTCTT GCCAGAAGTCTAGTGGTGGCCCTGAGGCGGCGGCATTCGTTAAAGTCTCCATGGACGGAGCGCCGTACCTGCGAAAGATTGATTTGCGAATGTATAAAAGTGGCGGCGGCGGCTCTTACCCGTACGAC GTCCCGGACTACGCTGGCTATCCCTATGATGTGCCCGATTATGCGTATCCTTACGATGTTCCAGATTATGCCTGATAACCGCCCACAGAAGC

\section{TEV-HA}

GGTGGAGGTAGCGGTGGTGGAAGTGAAAATCTGTACTTCCAGGGAGGTACCTACCCGTACGACGTCCCGGACTACGCTGGCTATCCCTATGATGTGCCCGATTATGCGTATCCTTACGATGTTCCAGAT TATGCCTGATAACCGCCCACAGAAGC

\section{TEV-GFP}

GGTGGAGGTAGCGGTGGTGGAAGTGAAAATCTGTACTTCCAGGGAGGTACCGCGGTGAGCAAGGGCGAGGAGCTGTTCACCGGGGTGGTGCCCATCCTGGTCGAGCTGGACGGCGACGTAAACGGC CACAAGTTCAGCGTGTCCGGCGAGGGCGAGGGCGATGCCACCTACGGCAAGCTGACCCTGAAGTTCATCTGCACCACCGGCAAGCTGCCCGTGCCCTGGCCCACCCTCGTGACCACCCTGACCTACG GCGTGCAGTGCTTCAGCCGCTACCCCGACCACATGAAGCAGCACGACTTCTTCAAGTCCGCCATGCCCGAAGGCTACGTCCAGGAGCGCACCATCTTCTTCAAGGACGACGGCAACTACAAGACCCGC GCCGAGGTGAAGTTCGAGGGCGACACCCTGGTGAACCGCATCGAGCTGAAGGGCATCGACTTCAAGGAGGACGGCAACATCCTGGGGCACAAGCTGGAGTACAACTACAACAGCCACAACGTCTATAT CATGGCCGACAAGCAGAAGAACGGCATCAAGGTGAACTTCAAGATCCGCCACAACATCGAGGACGGCAGCGTGCAGCTCGCCGACCACTACCAGCAGAACACCCCCATCGGCGACGGCCCCGTGCTG CTGCCCGACAACCACTACCTGAGCACCCAGTCCGCCCTGAGCAAAGACCCCAACGAGAAGCGCGATCACATGGTCCTGCTGGAGTTCGTGACCGCCGCCGGGATCACTCTCGGCATGGACGAGCTGTA CAAGTAGTCCTGATAACCGCCCACAGAAGC 
Table S3. List of proteins identified in the MS analysis of the GFP-Trap immunoprecipitated complexes of PfPanK1GFP- and PfPanK2-GFP-expressing parasites. Proteins are listed by the total number of peptides detected in the two independent replicates, from the most abundant to the least abundant. Only proteins that are present in the immunoprecipitation fractions of both parasite lines and absent in the negative controls (bound fractions of untagged GFPexpressing and 3D7 parasite lysates) are shown. Proteins shown in Figure 2a are indicated in red.

\begin{tabular}{|c|c|c|c|c|c|}
\hline \multirow{2}{*}{$\begin{array}{l}\text { Proteins detected in PfPanK1-GFP line } \\
\text { GFP-Trap immunoprecipitation }\end{array}$} & \multicolumn{2}{|c|}{$\begin{array}{l}\text { No. of peptides } \\
\text { (>95\% confidence) }\end{array}$} & \multirow{2}{*}{$\begin{array}{l}\text { Proteins detected in PfPanK2-GFP line } \\
\text { GFP-Trap immunoprecipitation }\end{array}$} & \multicolumn{2}{|c|}{$\begin{array}{l}\text { No of peptides } \\
\text { (>95\% confidence) }\end{array}$} \\
\hline & $1^{\text {st }}$ rep & $2^{\text {nd }}$ rep & & $1^{\text {st }}$ rep & $2^{\text {nd }}$ rep \\
\hline PfPanK1 & 20 & 23 & PfPanK2 & 16 & 77 \\
\hline PfPanK2 & 10 & 24 & PfPanK1 & 19 & 49 \\
\hline Pf14-3-3I & 7 & 14 & $P f 14-3-3 \mid$ & 16 & 41 \\
\hline M17 leucyl aminopeptidase & 3 & 4 & T-complex protein 1 subunit alpha & 4 & 14 \\
\hline elongation factor 1-gamma, putative & & 6 & elongation factor 2 & 3 & 14 \\
\hline heat shock protein $70-2$ & 3 & 2 & heat shock protein $70-2$ & 4 & 10 \\
\hline $26 \mathrm{~S}$ protease regulatory subunit 8 , putative & 1 & 2 & M17 leucyl aminopeptidase & 5 & 8 \\
\hline polyubiquitin & 1 & 2 & 40S ribosomal protein S11 & 3 & 6 \\
\hline ubiquitin-60S ribosomal protein $\mathrm{L} 40$ & 1 & 2 & elongation factor 1-gamma, putative & & 9 \\
\hline $60 S$ ribosomal protein L12, putative & 2 & 1 & glutamate--tRNA ligase, putative & 3 & 5 \\
\hline karyopherin beta & 2 & 1 & conserved Plasmodium protein (PF3D7_0813300) & 1 & 6 \\
\hline elongation factor 2 & & 3 & tubulin beta chain & 1 & 6 \\
\hline $26 \mathrm{~S}$ protease regulatory subunit 7 , putative & 1 & 1 & 60 S ribosomal protein $\mathrm{L} 12$, putative & 2 & 5 \\
\hline 265 protease regulatory subunit 4 , putative & 1 & 1 & $26 S$ protease regulatory subunit 8 , putative & & 7 \\
\hline endoplasmic reticulum-resident $\mathrm{Ca}^{2+}$ binding protein & 1 & 1 & ubiquitin-60S ribosomal protein $L 40$ & 3 & 3 \\
\hline plasmepsin IV & 1 & 1 & polyubiquitin & 3 & 3 \\
\hline tubulin beta chain & 1 & 1 & $40 S$ ribosomal protein $\mathrm{S} 25$ & 2 & 4 \\
\hline HSP40, subfamily A, putative & 2 & & $26 S$ protease regulatory subunit 7 , putative & & 6 \\
\hline protein DJ-1 & & 2 & karyopherin beta & 4 & 1 \\
\hline 40S ribosomal protein S5, putative & 1 & & $26 S$ protease regulatory subunit 4 , putative & & 5 \\
\hline 40 S ribosomal protein $\mathrm{S} 11$ & 1 & & 60 S ribosomal protein L13, putative & 1 & 3 \\
\hline $40 S$ ribosomal protein $\mathrm{S} 25$ & 1 & & $60 S$ ribosomal protein L18, putative & 1 & 3 \\
\hline 6-phosphofructokinase & 1 & & protein SIS1 & 1 & 3 \\
\hline $60 S$ ribosomal protein L18, putative & 1 & & HSP40, subfamily A, putative & 3 & 1 \\
\hline $60 S$ ribosomal protein $L 13$, putative & 1 & & phosphoethanolamine $\mathrm{N}$-methyltransferase & 1 & 4 \\
\hline $60 S$ ribosomal protein $L 23$, putative & 1 & & $40 S$ ribosomal protein S5, putative & & 5 \\
\hline DSK2, putative & 1 & & $40 S$ ribosomal protein $\mathrm{S} 20 \mathrm{e}$, putative & & 4 \\
\hline glutamate--tRNA ligase, putative & 1 & & 60 S ribosomal protein $L 23$, putative & 1 & 2 \\
\hline protein SIS1 & 1 & & 6-phosphofructokinase & 3 & \\
\hline $26 S$ proteasome regulatory subunit RPN8, putative & & 1 & 60S ribosomal protein $\mathrm{L} 1$, putative & & 3 \\
\hline 40S ribosomal protein S12, putative & & 1 & deoxyribose-phosphate aldolase, putative & 1 & 1 \\
\hline $40 S$ ribosomal protein $S 20 e$, putative & & 1 & endoplasmic reticulum-resident $\mathrm{Ca}^{2+}$ binding protein & 1 & 1 \\
\hline $60 S$ ribosomal protein $\mathrm{L} 1$, putative & & 1 & 26S proteasome regulatory subunit RPN8, putative & & 2 \\
\hline alpha tubulin 1 & & 1 & $40 S$ ribosomal protein $S 12$, putative & & 2 \\
\hline alpha tubulin 2 & & 1 & alpha tubulin 1 & & 2 \\
\hline conserved Plasmodium protein (PF3D7_0813300) & & 1 & alpha tubulin 2 & & 2 \\
\hline deoxyribose-phosphate aldolase, putative & & 1 & DSK2, putative & 1 & \\
\hline early transcribed membrane protein 10.2 & & 1 & early transcribed membrane protein 10.2 & & 1 \\
\hline phosphoethanolamine N-methyltransferase & & 1 & plasmepsin I & & 1 \\
\hline plasmepsin I & & 1 & plasmepsin IV & & 1 \\
\hline T-complex protein 1 subunit alpha & & 1 & protein DJ-1 & & 1 \\
\hline V-type proton ATPase catalytic subunit $A$ & & 1 & V-type proton ATPase catalytic subunit A & & 1 \\
\hline
\end{tabular}




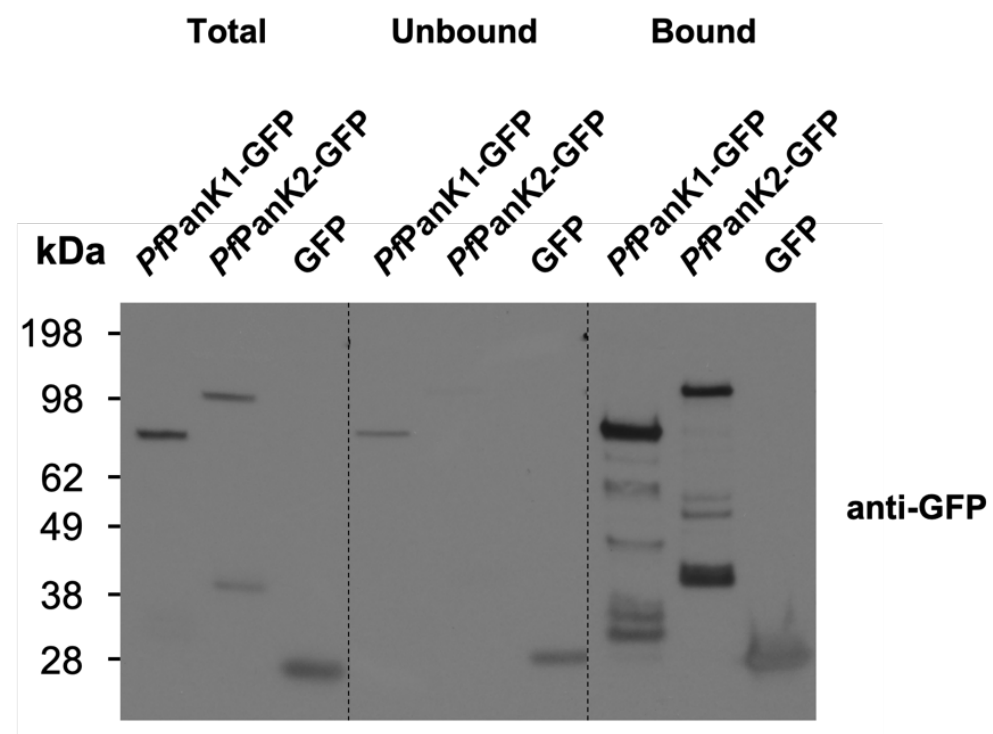

Figure S1. GFP-Trap immunoprecipitation of PfPanK1-GFP-, PfPanK2-GFP- and GFP-expressing parasites. Denaturing western blot analysis of the GFP-tagged proteins present in the total lysate, unbound and GFP-Trap-bound fractions of PfPanK1-GFP-, PfPanK2-GFP- and untagged GFP-expressing parasites. Western blots were performed with anti-GFP antibodies and the blot shown is representative of two independent experiments each performed with a different batch of parasites. 
bioRxiv preprint doi: https://doi.org/10.1101/2021.03.16.435557; this version posted March 16,2021 . The copyright holder for this preprint (which was not certified by peer review) is the author/funder, who has granted bioRxiv a license to display the preprint in perpetuity. It is made available under aCC-BY-NC-ND 4.0 International license.

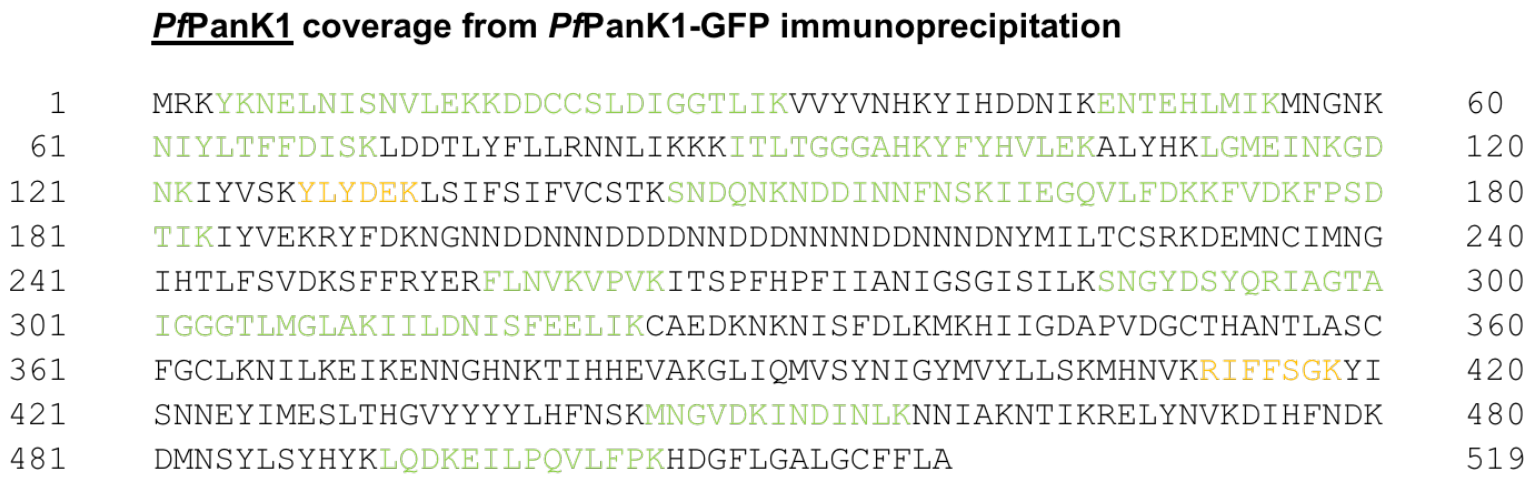

Coverage $=36 \%$

$\underline{P f P a n K 1}$ coverage from PfPanK2-GFP immunoprecipitation

1 MRKYKNELNISNVLEKKDDCCSLDIGGTLIKVVYVNHKYIHDDNIKENTEHLMIKMNGNK

60

NIYLTFFDISKLDDTLYFLLRNNLIKKKITLTGGGAHKYFYHVLEKALYHKLGMEINKGD 120

NKIYVSKYLYDEKLSIFSIEVCSTKSNDQNKNDDINNENSKIIEGQVLEDKKFVDKFPSD 180

TIKIYVEKRYFDKNGNNDDNNNDDDDNNDDDNNNNDDNNNDNYMILTCSRKDEMNCIMNG 240

IHTLFSVDKSFFRYERFLNVKVPVKITSPFHPFIIANIGSGISILKSNGYDSYQRIAGTA 300

IGGGTLMGLAKIILDNISEEEIIKCAEDKNKNISFDLKMKHIIGDAPVDGCTHANTLASC 360

FGCLKNILKEIKENNGHNKTIHHEVAKGLIQMVSYNIGYMVYLLSKMHNVKRIEFSGKYI 420

SNNEYIMESLTHGVYYYYLHFNSKMNGVDKINDINLKNNIAKNTIKRELYNVKDIHFNDK 480

DMNSYLSYYKLQDKEILPQVLFPKHDGFLGALGCFFLA 519

Coverage $=\mathbf{5 0} \%$

Figure S2. MS coverage of PfPanK1. PfPanK1 peptides detected in the two independent MS analyses of the GFP-Trap immunoprecipitation of the PfPanK1-GFP- and PfPanK2-GFP-expressing parasites. Residues in green were detected in either analysis with $>95 \%$ confidence, while residues in orange were detected in either analysis with $>90 \%$ (but $<95 \%$ ) confidence. Percentage coverage was calculated using only the residues labelled green. 
bioRxiv preprint doi: https://doi.org/10.1101/2021.03.16.435557; this version posted March $16,2021$. The copyright holder for this preprint (which was not certified by peer review) is the author/funder, who has granted bioRxiv a license to display the preprint in perpetuity. It is made available under aCC-BY-NC-ND 4.0 International license.

\title{
$\underline{\text { PfPanK2 }}$ coverage from PfPanK1-GFP immunoprecipitation
}

$\begin{array}{rll}1 & \text { MGNTLGIECSFNYVHVTTVLINKKLIKESNNDSKNEKDIREEKEKKLPKNVSIPSNDNKL } & 60 \\ 61 & \text { NMNVHLSWLKEKYKKEYINLEEDVSKSDEYNNCDDDYIKMKKNTFSYILDMQHIIDSDVQ } & 120 \\ 121 & \text { VFSLKKNSEKNVHTHISINELYKCFHEDDNLEKKFIKYLNFVKYHKLDISQIDTVNIQHL } & 180 \\ 181 & \text { YDDEIAEIYFWSFKLKYLDECILKYYKKNINYMFINVTGKNKLIKKKFLQITGKNNIFQ } & 240 \\ 241 & \text { HNEIKCINNSICFLKRFMPTNLYYFTKYNEENERASCTSQNDEDDEKKKKKKNLLYQSFI } & 300 \\ 301 & \text { NKEEVSEIKSYVIVNMKRAVCYHLVNEQNLIERIGTLYVGFRVMGLFLLITGRPCSLQR } & 360 \\ 361 & \text { ICQLAKNGTNRTFDMTVQDIYGTSYSNAGLCKDLTASFFGNAQHIENVKNILNTYDEEKN } & 420 \\ 421 & \text { INIKEEDDKLMNVYEYENESSCYENVSSCMSTEISECQEIFETEECIGFEVEKNNINYYR } & 480 \\ 481 & \text { NKYSFLSKDKTKKLLVSNKNENVYDNCKIPIFNFNSDSYCNYMNSFKSKEKLFKHNIEEQ } & 540 \\ 541 & \text { OKQEEGYKQKEDDNFLMNDIDNYLSIKHSLSDNEINMYEYHRKNYNLKKSFLKKLFKKQY } & 600 \\ 601 & \text { LNENGKIIHNINLSIQKEVSSEILKNKKRFSYKTYDKQIVKKACKSITLKNVCSETNKNK } & 660 \\ 661 & \text { MDSNLKDKTIVKYNTKMCDLSRSLLSMAIFTTVYLSYIHCNLYNADHIFFTGYNFEDDVC } & 720 \\ 721 & \text { KELFQIIIKFLSHNRQKIYFAKISKYISSLGSAIELINWERIHIDN } & 766\end{array}$

Coverage $=29 \%$

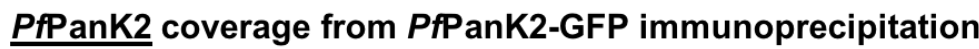

\begin{abstract}
MGNTLGIECSFNYVHVTTVLINKKLIKESNNDSKNEKDIREEKEKKLPKNVSIPSNDNKL NMNVHLSWLKEKYKKEYINLEEDVSKSDEYNNCDDDY IKMKKNTFSY ILDMQH I IDSDVQ VFSLKKNSEKNVHTHISINELYKCFHEDDNLEKKFIKYLNFVKYHKLDISQIDTVNIQHL YDDEIAEIYFWSFKLKYLDECILKYYKKNINYMFINVTGKNKNLIKKKFLQITGKNNIFQ HNEIKCINNS ICFLKREMP TNLYYFTKYNEENERASCTSQNDEDDEKKKKKKNLLYQSEI NKEEVSEIKSYVIVNMKRAVCYHLVNEQNLIERIGTLYVGERTVMGLFLLITGRPCSLQR ICQLAKNGTNRTFDMTVQDIYGTSYSNAGLCKDLTASFFGNAQHIENVKNILNTYDEEKN INIKEEDDKLMNVYEYENESSCYENVSSCMSTEISECQEIFETEECIGFEVEKNN INYYR NKYSFLSKDKTKKLLVSNKNFNVYDNCKIPI FNFNSDSYCNYMNSFKSKEKLFKHNIEEQ QKQEEGYKQKEDDNFLMND I DNYLS I KHSLSDNE INMYEYHRKNYNLKKSELKKLFKKQY LNENGKI IHNINLS IQKEVSSFILKNKKRFSYKTYDKQIVKKACKS ITLKNVCSETNKNK MDSNLKDKTIVKYNTKMCDLSRSLLSMAIFTTVYLSYI HCNLYNADH IFFTGYNFEDDVC KELFQIIIKFLSHNRQKIYFAKISKYISSLGSAIELINWERIHIDN
\end{abstract}

60

120

180

240

300

360

420

480

540

600

660

720

766

\section{Coverage $=49 \%$}

Figure S3. MS coverage of PfPanK2. PfPanK2 peptides detected in the two independent MS analyses of the GFP-Trap immunoprecipitation of the PfPanK1-GFP- and PfPanK2-GFP-expressing parasites. Residues in green were detected in either analysis with $>95 \%$ confidence, while residues in orange were detected in either analysis with $>90 \%$ (but $<95 \%$ ) confidence. Percentage coverage was calculated using only the residues labelled green. 
bioRxiv preprint doi: https://doi.org/10.1101/2021.03.16.435557; this version posted March 16,2021 . The copyright holder for this preprint (which was not certified by peer review) is the author/funder, who has granted bioRxiv a license to display the preprint in perpetuity. It is made available under aCC-BY-NC-ND 4.0 International license.

\section{$\underline{P f 14-3-31}$ coverage from PfPanK1-GFP immunoprecipitation}

$\begin{array}{rll}1 & \text { MATSEELKQLRCDCTYRSKLAEQAERYDEMADAMRTLVEQCVNNDKDELTVEERNLLSVA } & 60 \\ 61 & \text { YKNAVGARRASWRIISSVEQKEMSKANVHNKNVAATYRKKVEEELNNICQDILNLLTKKL } & 120 \\ 121 & \text { IPNTSESESKVFYYKMKGDYYRYISEFSCDEGKKEASNCAQEAYQKATDIAENELPSTHP } & 180 \\ 181 & \text { IRLGLALNYSVFFYEILNQPHQACEMAKRAFDDAITEFDNVSEDSYKDSTLIMQLLRDNL } & 240 \\ 241 & \text { TLWTSDLQGDQTEEKSKDEGLE } & 262\end{array}$

Coverage $=43 \%$

\section{$\underline{P f 14-3-31}$ coverage from PfPanK2-GFP immunoprecipitation}

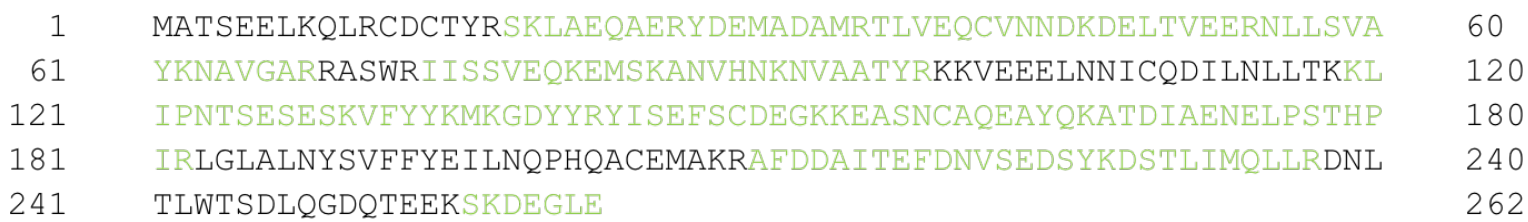

Coverage $=67 \%$

Figure S4. MS coverage of Pf14-3-3I. Pf14-3-3I peptides detected in the two independent MS analyses of the GFP-Trap immunoprecipitation of the PfPanK1-GFP- and PfPanK2-GFP-expressing parasites. Residues in green were detected in either analysis with $>95 \%$ confidence, while residues in orange were detected in either analysis with $>90 \%$ (but $<95 \%$ ) confidence. Percentage coverage was calculated using only the residues labelled green. 
a

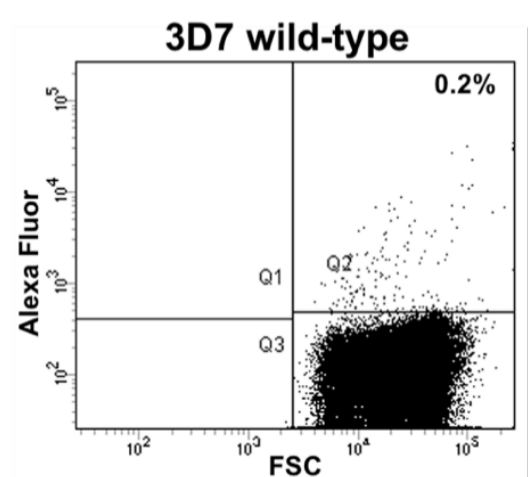

b

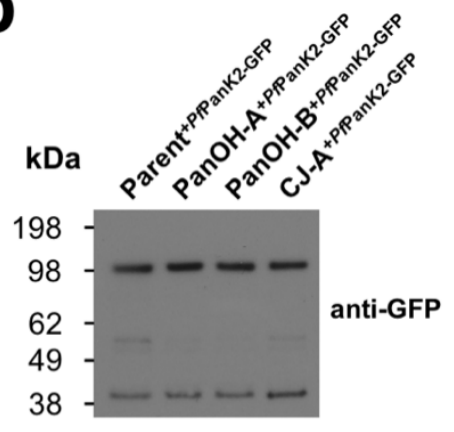

Experiment 1
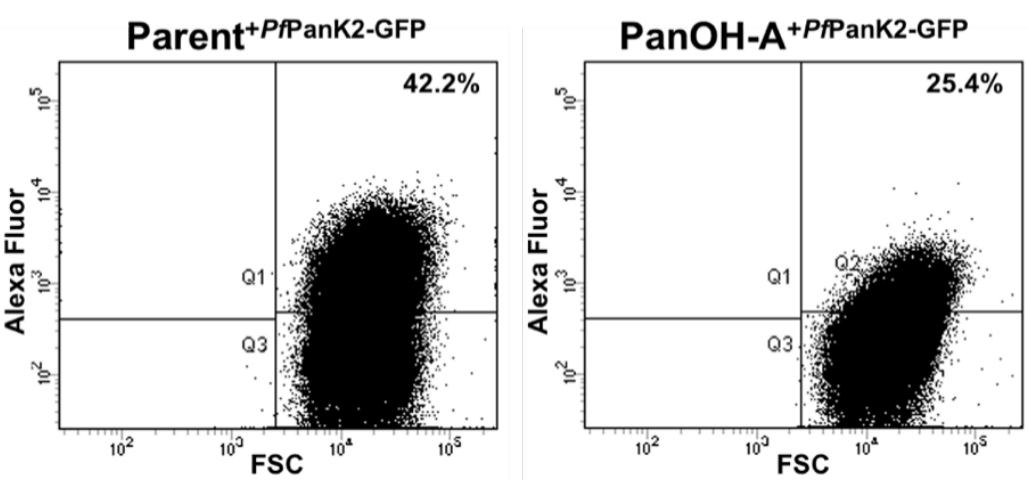

PanOH-B + PfPanK2-GFP
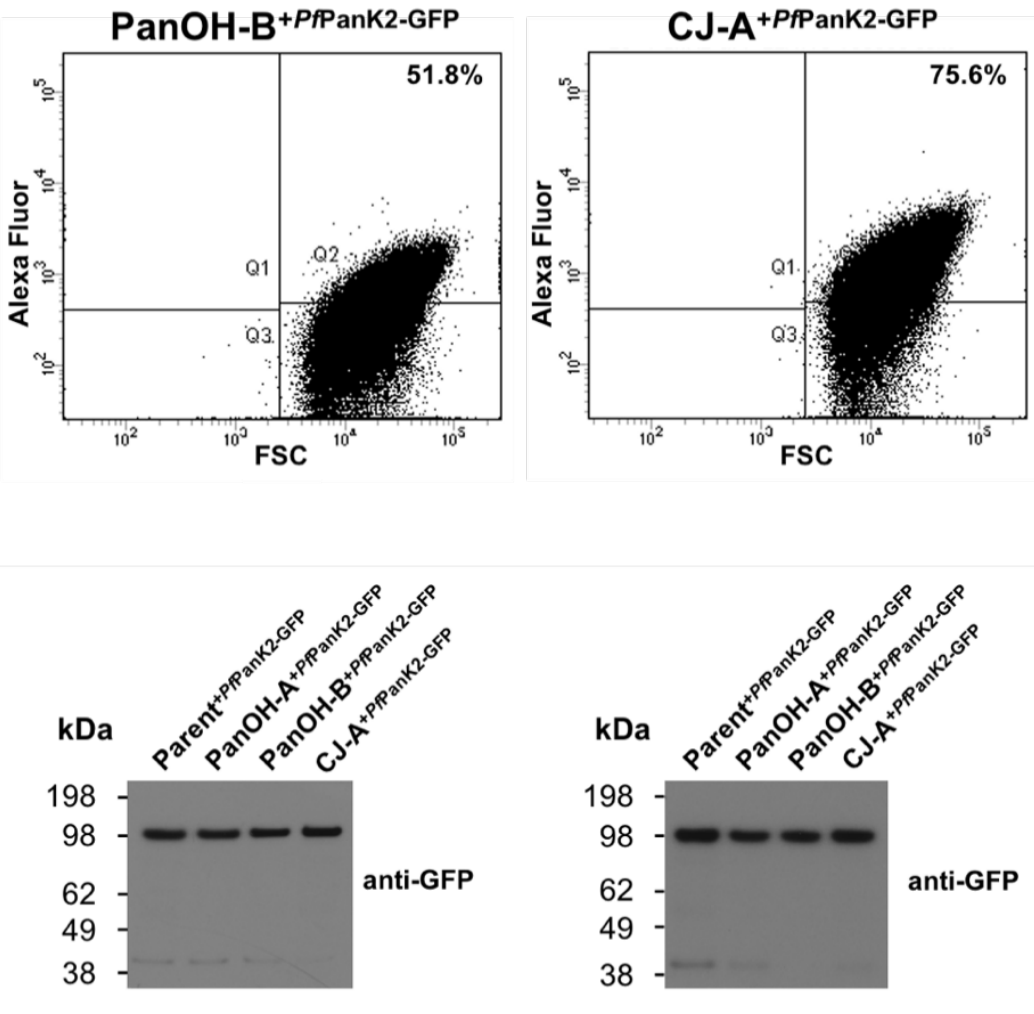

Experiment 2

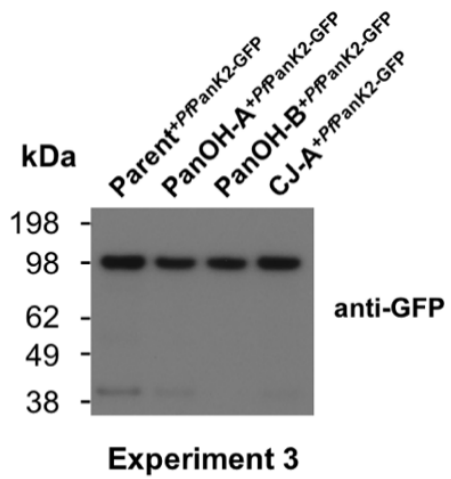

Figure S5. Determining the amount of GFP-Trap bound PfPanK2-GFP for pantothenate phosphorylation assays. (a) The proportion of GFP-positive saponin-isolated 3D7, Parent ${ }^{+P \text { IPank2-GFP, }}$ PanOH-A ${ }^{+P \text { PPanK2-GFP }}$, PanOH-B ${ }^{+P \text { PPanK2-GFP }}$ and CJ-A ${ }^{+P \text { PPanK2-GFP }}$ trophozoites was determined by FACS analysis. The forward scatter (FSC) intensity on each $x$-axis corresponds to cell size and the AlexaFluor intensity on each y-axis corresponds to GFP fluorescence. The proportion of GFP-positive cells in each transgenic line (percentage value in each plot) was determined by using 3D7 trophozoites to set a gating threshold below which parasites were defined to be auto-fluorescent. Data shown are representative of three independent experiments, each performed prior to the $\left[{ }^{14} \mathrm{C}\right]$ pantothenate phosphorylation assays presented in Figure $2 \mathrm{bii}$. The flow cytometry data was used to standardise the amount of PfPanK2-GFP immunoprecipitated from each cell line used in each $\left[{ }^{14} \mathrm{C}\right]$ pantothenate phosphorylation assay. (b) Denaturing western blot analysis of PfPanK2-GFP in the GFP-Trap immunoprecipitated complexes that were used in the $\left[{ }^{14} \mathrm{C}\right]$ pantothenate phosphorylation assays performed to generate the data in Figure $2 \mathrm{~b}$. Western blots were performed with an anti-GFP antibody and each blot shows the relative amounts of PfPanK2-GFP immunopurified from the four different cell lines used in each of the three $\left[{ }^{14} \mathrm{C}\right]$ pantothenate phosphorylation experiment. The same volume of samples $(10 \mu \mathrm{L}$ per lane) was used for all three experiments. 
DNORTFKTELTKNIDOVVEWLNOO

OIEKLCLTGGNAGVIAENIN

56

21 FNLAIDIGGTLAKVVFSP [ 2] SNRLMFYTIETEKIDKFMELLHSI IKEHN--------NGCYRMTHIIATGGGAFKFYDLLYENF-----PQIKGISRFE

(237

HSPanK2 212 PWFGIDIGGTVVKVYFE [ 49]

---NVDIMRED

HsPanK3

PWFGMDIGGTLVKLSYFE [ 49] RGNLHFIRFPTQDLPTFIQMGRDKN--..--NLHLHKL

HsPanK4

35 KRFAIDIGGSLTKLAYYS [ 35] TARLHFIKFENTYIEACLDFIKDHL-----------VNTETKVIQATGGGAYKFKDLIEEKL SHLALDIGGTLIKLVYFS [ 16] KGRLCFAKFETRKIDDCLEFIRFNI LHHSGVQQ--PNGEGHDKLYVKATGGGAFKFADLFKEKL-

AtPank

AtPank2

PfPanK1

SHLALDIGGSLIKLLYFS [ 33] GGRLHFVKFETHKINECLDF IHSKQLHRRDPYPWSSKTLPLGTGVIKVTGGGAFKFADLFKERL

19 DCCSLDIGGTLIKVVYVN [ 22] NKNIYLTFFDISKLDDTLYFLLRNN-------------LIKKKITLTGGGAHKYFYHVLEKA []

TgPanK1 358 DRCAVDIGGTLAKVVFVT[247] EHVIRFTYFHTKDVSSLLHFLKANG--------- FAQPGVVLRATGGGAHKFSALFLERI

TgPanK2

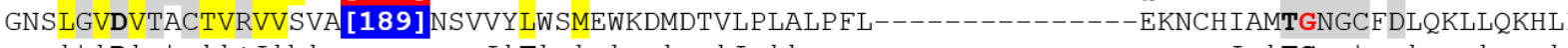

Consensus AA:

Consensus sS:

Conservation:

SaPanK-II 86

SCPanK-II 145

AnPanK-II 193

HsPanK1

HsPanK2

HsPanK3

HsPanK3

Hs PanK

AtPanK1

AtPank2

PfPanK2

TgPanK

TgPanK2

$h$ thDhstshh+Lhbh

$$
\begin{aligned}
& \text { F F } \\
& \text { F } h \text {. }
\end{aligned}
$$

TGGGA $\mathrm{K}$

\section{DIGGTL $\mathrm{K}$ \\ eeeee \\ eeeeeee \\ eeeeeee hhhhhhhhhh}

-

[115]

-GILFDKE

[156]

\section{- - PHOSPHATE 2}

$6 \quad 5,7975767 \quad 5$

hhhhhhhhhhh 
Figure S6. Multiple sequence alignment of representative Type II PanKs. The conserved PHOSPHATE 1, PHOSPHATE 2, and ADENOSINE 1 motifs of the acetate and sugar kinases/Hsc70/actin (ASKHA) superfamily of kinases are labelled at the top of the alignment. The Glu (E) residue involved in catalysis and the Arg $(R)$ residue involved in positioning the substrate, are shown on a black background. Residues that have been found to interact with pantothenate and acetyl-CoA in human PanK $3^{9,10}$ are marked with a blue asterisk. Residues that were found to interact to stabilise the human PanK3 active site are marked with a red asterisk. The catalytic Glu (E) residue is marked with a red and blue asterisk as it is involved in both the interaction with pantothenate and the stabilisation of the active site through interaction with a $\operatorname{Tyr}(\mathrm{Y})$ residue of the opposite protomer. The numbers at the start and end of each sequence indicate the position of the first and last residue in the alignment, respectively. The lengths of insertions are specified within the square brackets and the total length of protein sequences are shown in round brackets. Residues within the ASKHA superfamily motifs and conserved residues are highlighted based on the consensus AA guide for the column as follows: identical $=$ bold, hydrophobic (W,F,Y,M ,L,I,V,A,C,T,H) = yellow, charged/polar/small (D,E,K,R,H/D,E,H,K,N,Q,R,S,T/A,G,C,S,V,N,D,T,P) = grey and Gly = red. The two insertion regions (Ins 1 and Ins 2) common to eukaryotic type II PanKs, but absent in prokaryotic PanKs are indicated by the black horizontal bars, while the PfPank1/TgPanK1 and PfPanK2/TgPanK2 specific inserts are highlighted on a red and blue background, respectively. Conservation refers to the conservation indices. Values at and above the conservation index cut-off (5) are displayed above the amino acid. Consensus AA: refers to the consensus level alignment parameters for the consensus amino acid sequence. This is displayed if the weighted frequency of a certain class of residues in a position is above 0.8 . Consensus symbols: conserved amino acids are in bold and uppercase letters; aliphatic (I, V, L): I; aromatic (Y, H, W, F): @; hydrophobic (W, F, Y, M, L, I, V, A, C, T, H): $h$; alcohol (S, T): o; polar residues (D, E, H, K, $\mathrm{N}, \mathrm{Q}, \mathrm{R}, \mathrm{S}, \mathrm{T})$ : p; tiny $(\mathrm{A}, \mathrm{G}, \mathrm{C}, \mathrm{S})$ : t; small $(\mathrm{A}, \mathrm{G}, \mathrm{C}, \mathrm{S}, \mathrm{V}, \mathrm{N}, \mathrm{D}, \mathrm{T}, \mathrm{P})$ : s; bulky residues $(\mathrm{E}, \mathrm{F}, \mathrm{I}, \mathrm{K}, \mathrm{L}, \mathrm{M}, \mathrm{Q}, \mathrm{R}, \mathrm{W}, \mathrm{Y})$ : b; positively charged $(\mathrm{K}, \mathrm{R}, \mathrm{H})$ : +; negatively charged (D, E): -; charged (D, E, K, R, H): c. Marked below the alignment, $85 \%$ consensus includes those residues that occur in either the superfamily motifs and/or conserved residues where the same residue occurs more than $85 \%$ (10 out of 13 sequences). Consensus secondary structure (ss) elements: $\mathrm{h}=\mathrm{alpha}$ helix, e $=$ beta strand. Species names are abbreviated as follows: $\mathrm{Sa}=$ Staphylococcus aureus, Sc $=$ Saccharomyces cerevisiae, An = Aspergillus nidulans, $\mathrm{Hs}=\mathrm{Homo}$ sapiens, $\mathrm{At}$ $=$ Arabidopsis thaliana, $P f=$ Plasmodium falciparum and $T g=$ Toxoplasma gondii. The alignment was created using PROMALS3D ${ }^{8}$. 
a
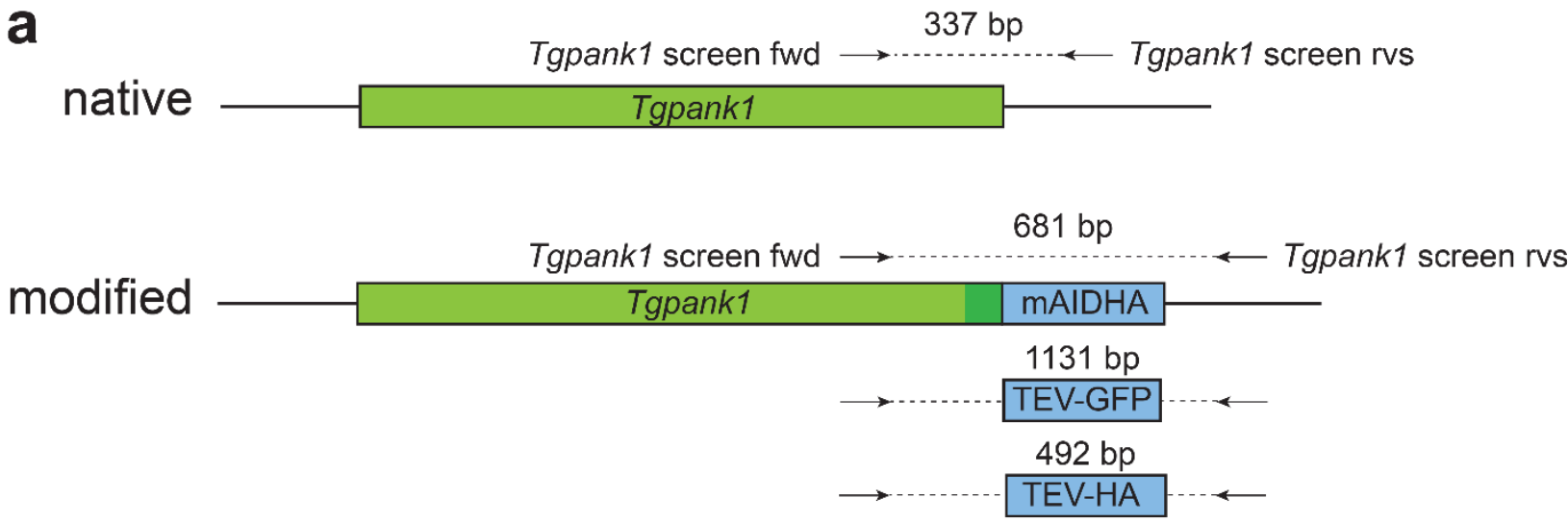

$267 \mathrm{bp}$

native

Tgpank2 screen fwd

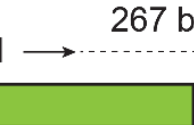

$611 \mathrm{bp}$

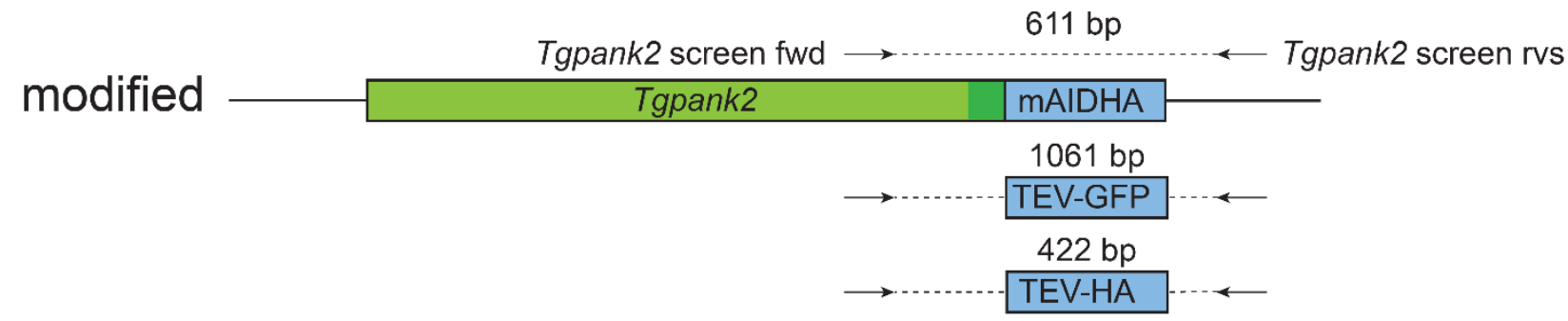

b

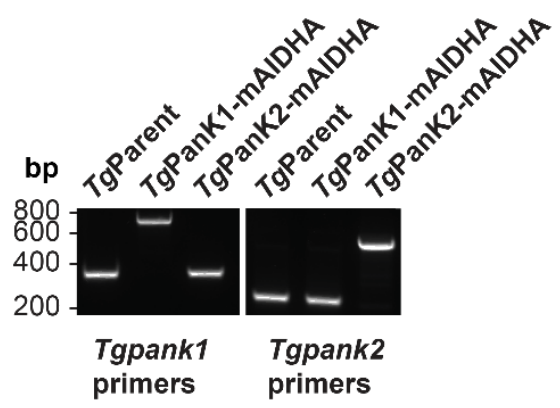

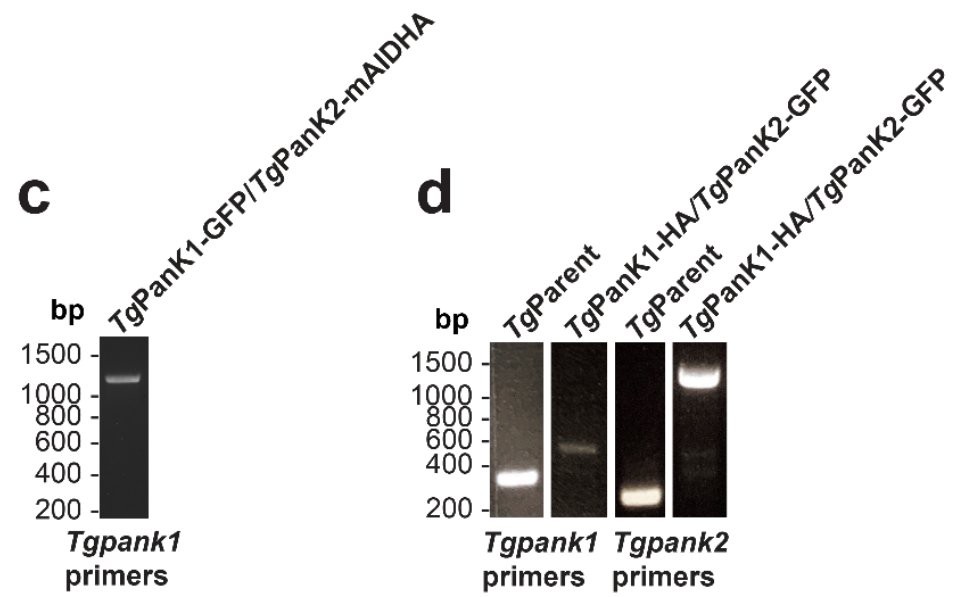

Figure S7 Gene models and confirmation of the incorporation of the coding sequence for various epitope tags into the Tgpank1 and Tgpank2 loci. (a) Gene models for Tgpank1 and Tgpank2 indicating the incorporation site of the epitope tag coding sequence. The expected sizes of the PCR products when screened with each set of screening primers are shown above the corresponding epitope tag coding sequence. The screening primers are Tgpank1 screen fwd and rev for Tgpank1 (referred to as Tgpank1 primers in the panels b-d), and Tgpank2 screen fwd and rev for Tgpank2 (referred to as Tgpank2 primers in panels b-d). Primers are detailed in Table S1. (b) PCR analysis of the TgParent, and singly-tagged TgPanK1-mAIDHA and TgPanK2-mAIDHA clonal lines. As can be seen, both TgPanK1-mAIDHA and TgPanK2-mAIDHA have successfully incorporated mAIDHA tags. (c) PCR analysis of the doubly-tagged TgPanK1-GFP/TgPanK2mAIDHA clonal line. CRISPR/Cas9 was utilised to incorporate a sequence encoding a TEV-GFP tag into the genomic locus of the Tgpank1 gene within the TgPanK2-mAIDHA-expressing line. (d) PCR analysis of the TgPanK1-HA/TgPanK2-GFP doubly tagged clonal line (Tg Clone B4C6). CRISPR/Cas9 was utilised to incorporate a sequence encoding a TEV-HA tag into the genomic locus of the Tgpank1 gene and a sequence encoding TEV-GFP tag into the genomic locus of the Tgpank2 gene. 


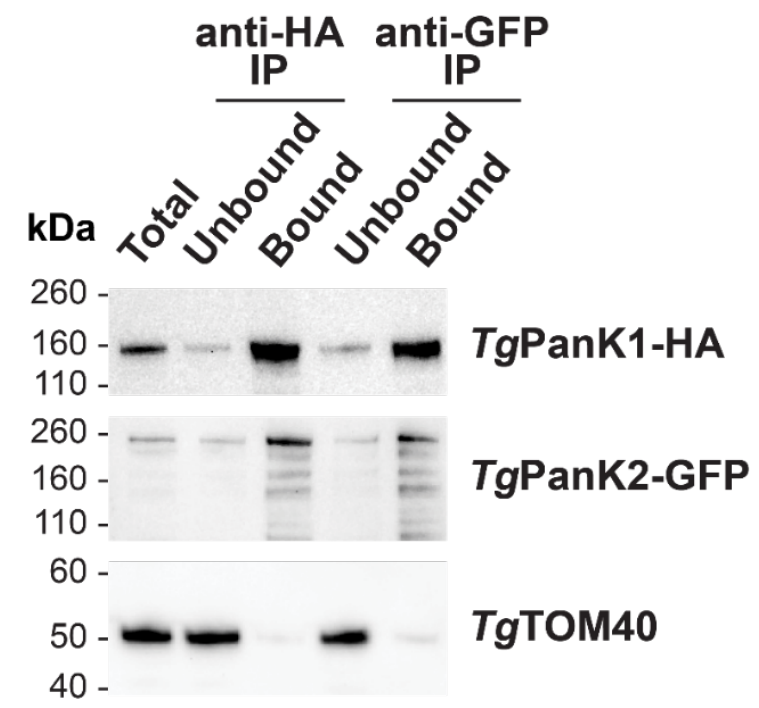

Figure S8. Anti-GFP and anti-HA immunoprecipitation of TgPanK1-HA/TgPanK2-GFP expressing parasites. Anti-HA and anti-GFP denaturing western blot analysis of fractions from GFP-Trap and anti-HA immunoprecipitations performed using lysates prepared from the parasite lines expressing TgPanK1HA/TgPanK2-GFP. The expected molecular masses of TgPanK1 and TgPanK2 are $\sim 132 \mathrm{kDa}$ and $\sim 178 \mathrm{kDa}$, respectively. The molecular mass of GFP is $\sim 27 \mathrm{kDa}$. The blot shown is representative of three independent experiments, each performed with different batches of parasites. Denaturing western blots were also probed with anti-TgTOM40, which served as a loading control. 

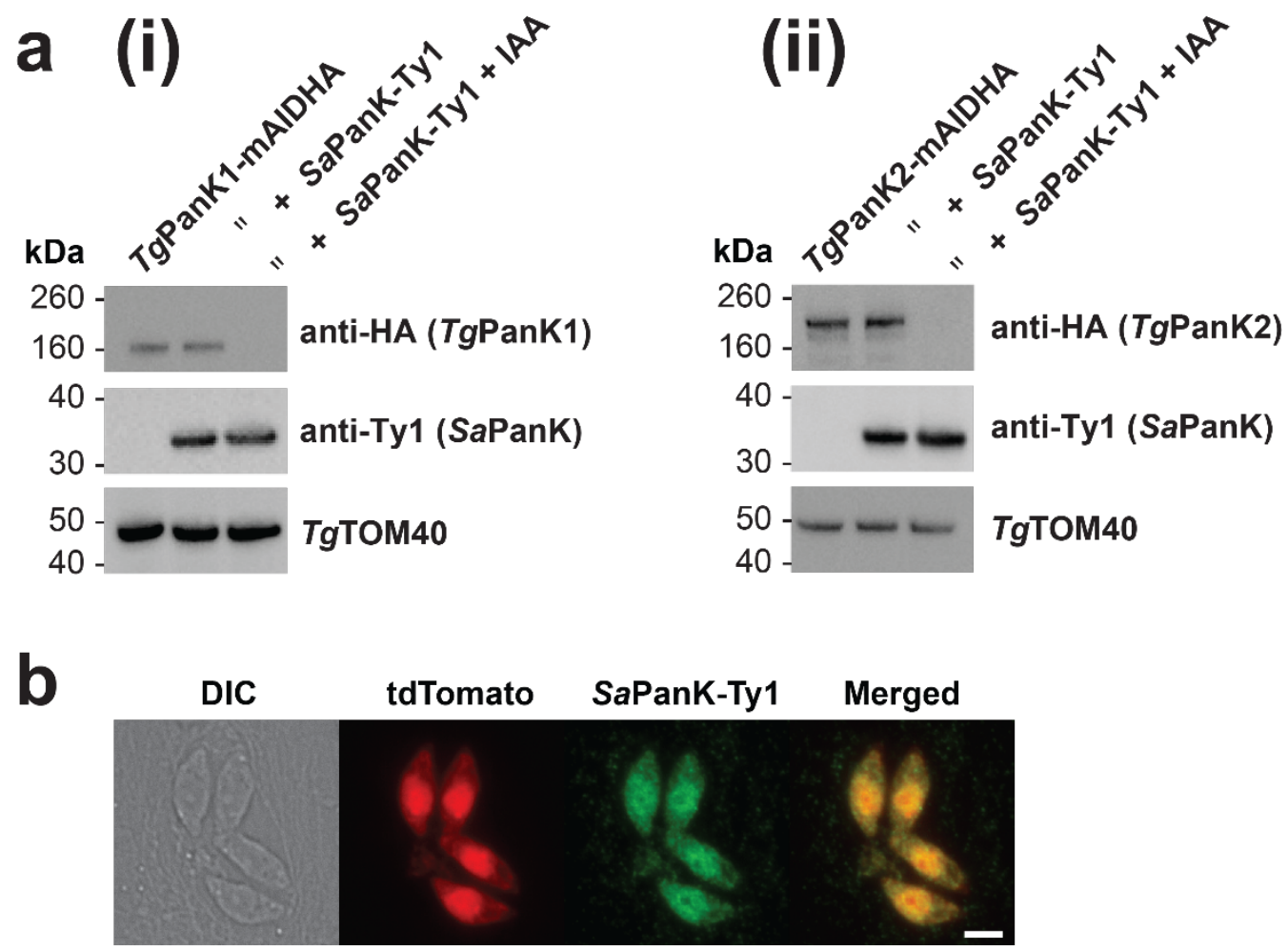

Figure S9. Expression of SaPanK-Ty1 in TgPanK1-mAIDHA- and TgPanK2-mAIDHA-expressing parasites. (a) Anti-HA and anti-Ty1 denaturing western blot analysis of SaPanK-Ty1-complemented and noncomplemented (i) TgPanK1-mAIDHA- and (ii) TgPanK2-mAIDHA-expressing lines, in the absence or presence (for $1 \mathrm{~h}$ ) of $100 \mu \mathrm{M}$ IAA. The expected molecular masses of TgPanK1-mAIDHA, TgPanK2-mAIDHA and SaPanK-Ty1 are $\sim 141 \mathrm{kDa}, \sim 187 \mathrm{kDa}$ and $\sim 29 \mathrm{kDa}$, respectively. Denaturing western blots were also probed with anti-TgTOM40, which served as a loading control. Each blot shown is representative of three independent experiments, each performed with a different batch of parasites. (b) Fluorescence micrographs of a HFF cell infected with four tachyzoite-stage TgPanK1-mAIDHA ${ }^{+S a P a n K-T y 1}$ parasites within a vacuole, indicating the presence of SaPanK-Ty1. From left to right: Differential interference contrast (DIC), tdTomato-fluorescence indicating the location of the parasites within the host cell, anti SaPanK-Ty1 AlexaFluor 488 fluorescence, and merged images. Scale bar represents $2 \mu \mathrm{m}$. 


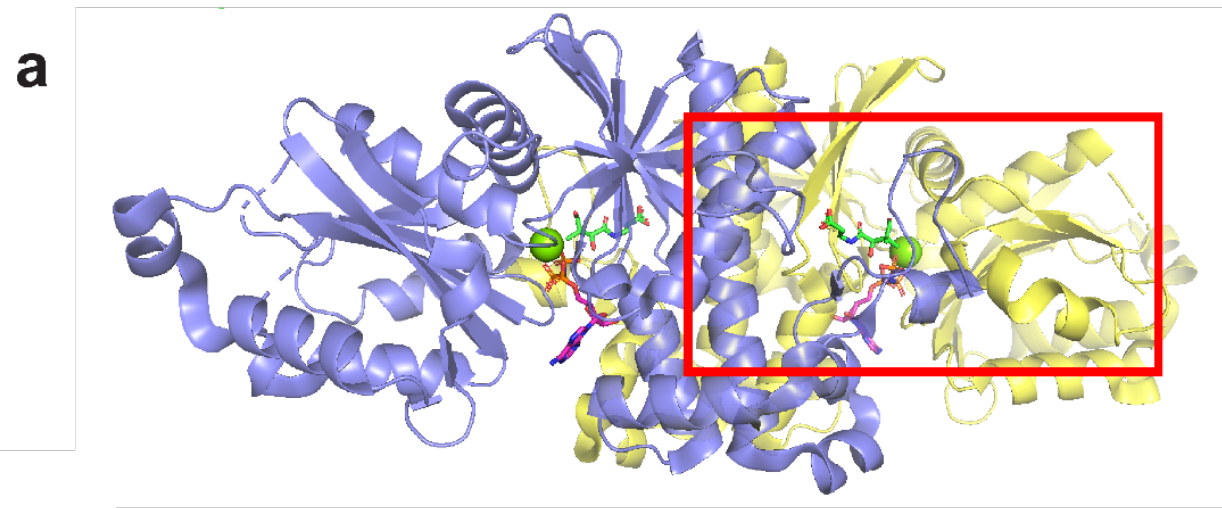

b

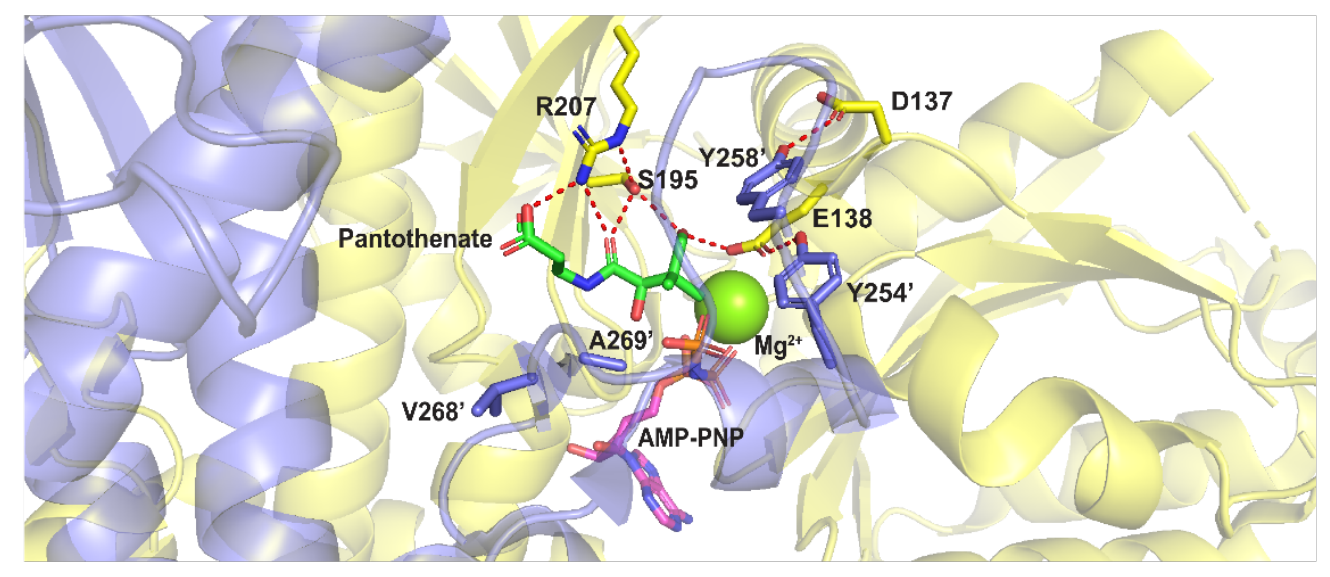

C

\begin{tabular}{|c|c|c|c|c|}
\hline HsPanK3 & PfPanK1 & PfPanK2 & TgPanK1 & TgPanK2 \\
\hline D137 & D232 & N242 & D206 & $\bar{R} 424$ \\
\hline E138 & E233 & E243 & E207 & E425 \\
\hline Y258' & 1343 & Y381 & F315 & Y742 \\
\hline Y254' & P347 & Y385 & A319 & Y746 \\
\hline
\end{tabular}

Figure S10. Pantothenate binding site and interactions in $\boldsymbol{H}$. sapiens PanK3. (a) H. sapiens AMP-PNPpantothenate-bound PanK3 crystal structure (PDB ID: 5KPR, Subramanian et al. ${ }^{10}$ ). The homodimeric protein is made up of two identical protomers (lilac and yellow) forming two identical active sites, each binding pantothenate (green). The red square encompasses one of the active sites. (b) Magnification of the region outlined by the red square in (a). Residues from both protomers contribute to the stabilisation of the binding pocket (E138 forms a hydrogen bond with Y254' and D137 with Y258') and interact with pantothenate (E138, S195, R207, A269' and V268'). Hydrogen bonds with and between the sidechains of these residues are shown in red. An apostrophe denotes residues from the lilac protomer. (c) List of residues annotated in the HsPanK3 model that participate in the stabilisation of the binding pocket (highlighted cyan), and a comparison to the equivalent residues in $P$. falciparum and $T$. gondii PanKs. The PanKs from $P$.

falciparum and $T$. gondii do not individually contain the complete set of residues required for the stabilisation of the binding pocket, but the combination of residues (highlighted cyan) from PanK1 and PanK2 suggests that each PanK1/PanK2 heterodimer will have only one stabilised binding site. 
bioRxiv preprint doi: https://doi.org/10.1101/2021.03.16.435557; this version posted March 16,2021 . The copyright holder for this preprint (which was not certified by peer review) is the author/funder, who has granted bioRxiv a license to display the preprint in perpetuity. It is made available under aCC-BY-NC-ND 4.0 International license.

\section{Apicomplexa}

\section{Haematozoa}

Plasmodium falciparum

Plasmodium berghei

Babesia microti

Theileria annulata

Theileria parva

\section{Coccidia}

Toxoplasma gondii (GT1)

Toxoplasma gondii (ME49)

Sarcocystis neurona

Neospora caninum

Hammondia hammondi

Eimeria tenella

Cryptosporidium parvum

Cryptosporidium hominis

Gregarines

Gregarina niphandrodes

\section{PanK1}

HsPanK3 D137 and E138 conserved

HsPanK3 Y254 and Y258 not conserved
PanK2

HsPanK3 Y254 and Y258 conserved HsPanK3 D137 and E138 not conserved

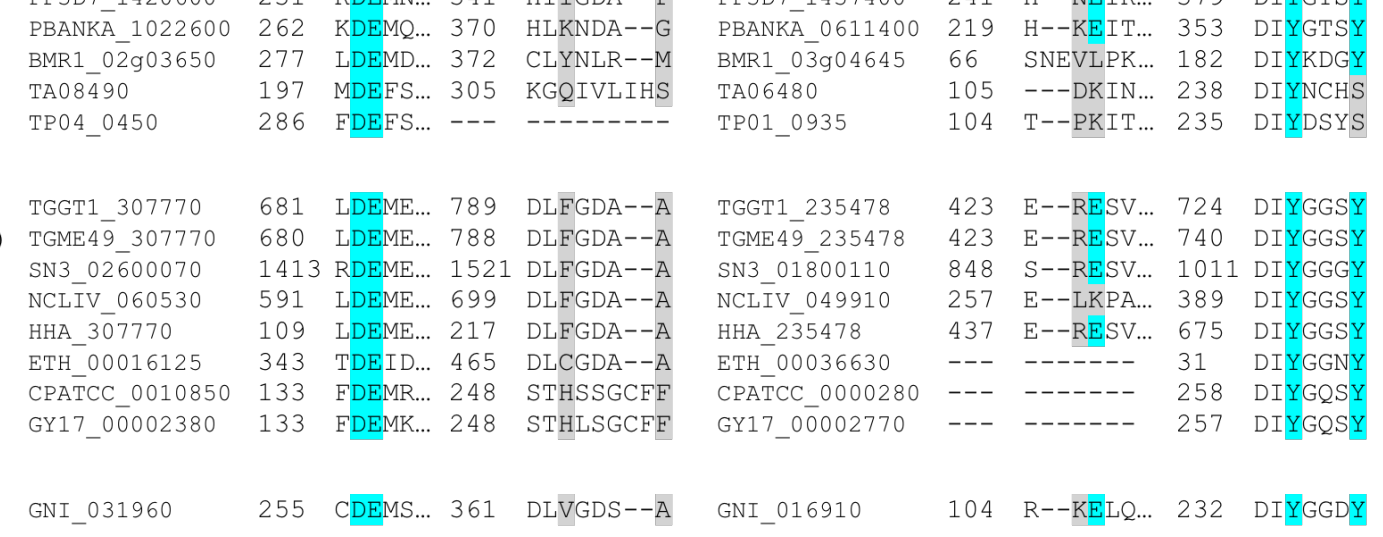$$
7 \text { I }
$$$$
7 \mathrm{MDE}
$$

GNI_031960

255 CDEMS... 361 DLVGDS--A

GN1_ 016910

104 R--KELQ... 232 DIYGGDY

Figure S11. Multiple sequence alignment of active site stabilisation residues in apicomplexan PanKs. The apicomplexan PanK residues corresponding to the HsPanK3 residues that are involved in the stabilisation of the binding pocket (D137, E138, Y254 and Y258) are highlighted in cyan if they are conserved and in grey if they are not conserved. The numbers before each alignment indicate the position of the first residue in the alignment. Each apicomplexan PanK is grouped into PanK1 or PanK2 based on their similarity to either PfPanK1/TgPanK1 or PfPanK2/TgPanK2, respectively. The alignment was created using PROMALS3D ${ }^{8}$. 


\section{SUPPLEMENTARY REFERENCES}

1. Shen, B., Brown, K. M., Lee, T. D. \& Sibley, L. D. Efficient gene disruption in diverse strains of Toxoplasma gondii using CRISPR/CAS9. MBio 5, e01114-14 (2014).

2. Brown, K. M., Long, S. \& Sibley, L. D. Plasma Membrane Association by N-Acylation Governs PKG Function in Toxoplasma gondii. MBio 8, F1000 (2017).

3. Brooks, C. F. et al. The toxoplasma apicoplast phosphate translocator links cytosolic and apicoplast metabolism and is essential for parasite survival. Cell Host Microbe 7, 62-73 (2010).

4. Rajendran, E. et al. Cationic amino acid transporters play key roles in the survival and transmission of apicomplexan parasites. Nat. Commun. 8, 14455 (2017).

5. Messina, M., Niesman, I., Mercier, C. \& Sibley, L. D. Stable DNA transformation of Toxoplasma gondii using phleomycin selection. Gene 165, 213-217 (1995).

6. Bastin, P., Bagherzadeh, Z., Matthews, K. R. \& Gull, K. A novel epitope tag system to study protein targeting and organelle biogenesis in Trypanosoma brucei. Mol. Biochem. Parasitol. 77, 235-239 (1996).

7. Gubbels, M.-J., Li, C. \& Striepen, B. High-throughput growth assay for Toxoplasma gondii using yellow fluorescent protein. Antimicrob. Agents Chemother. 47, 309-316 (2003).

8. Pei, J. \& Grishin, N. V. PROMALS3D: multiple protein sequence alignment enhanced with evolutionary and three-dimensional structural information. Methods Mol. Biol. 1079, 263-271 (2014).

9. Leonardi, R. et al. Modulation of pantothenate kinase 3 activity by small molecules that interact with the substrate/allosteric regulatory domain. Chem. Biol. 17, 892-902 (2010).

10. Subramanian, C. et al. Allosteric regulation of mammalian pantothenate kinase. J. Biol. Chem. 291, 22302-22314 (2016). 